\title{
Vorticity budgets in premixed combusting turbulent flows at different Lewis numbers
}

Dopazo, Cesar; Cifuentes, Luis; Chakraborty, Nilanjan

This text is provided by DuEPublico, the central repository of the University Duisburg-Essen.

This version of the e-publication may differ from a potential published print or online version.

DOI: https://doi.org/10.1063/1.4981219

URN: urn:nbn:de:hbz:464-20190118-084604-4

Link: https://duepublico.uni-duisburg-essen.de:443/servlets/DocumentServlet?id=47966

Legal notice:

This article may be downloaded for personal use only. Any other use requires prior permission of the authors and AIP Publishing.

Source: This article appeared in Physics of Fluids 29, 045106 (2017) and may be found at https://doi.org/10.1063/1.4981219 published online 28 April 2017 


\title{
Vorticity budgets in premixed combusting turbulent flows at different Lewis numbers
}

\author{
Cesar Dopazo, ${ }^{1}$ Luis Cifuentes, ${ }^{1,2, a)}$ and Nilanjan Chakraborty ${ }^{3}$ \\ ${ }^{1}$ School of Engineering and Architecture - Fluid Mechanics Area, University of Zaragoza, C/ Maria de Luna 3, \\ Zaragoza 50018, Spain \\ ${ }^{2}$ Institute for Combustion and Gasdynamics (IVG), Chair for Fluid Dynamics, University of Duisburg-Essen, \\ Duisburg 47048, Germany \\ ${ }^{3}$ School of Mechanical and Systems Engineering, Newcastle University, Claremont Road, \\ Newcastle-Upon-Tyne NE1 7RU, United Kingdom
}

(Received 19 October 2016; accepted 5 April 2017; published online 28 April 2017)

\begin{abstract}
A direct numerical simulations database of statistically planar turbulent premixed flames using a simple Arrhenius type irreversible chemistry for different values of global Lewis numbers, $L e,(0.34$, $0.60,0.80,1.00,1.20)$ has been examined to analyze the effects of $L e$ on vorticity transport within the flame. To meet this objective, a general enstrophy conservation equation has been considered, which distinctly describes contributions from vortex-stretching, destruction by volumetric dilatation rates, baroclinic and viscous force torques, viscous transport, and dissipation. The average statistical behavior of the various contributions conditioned upon the value of the reaction progress variable, $c$, has been analyzed in the preheat and reacting regions of the flame. The mean values of enstrophy monotonically decays with $c$ from fresh reactants toward hot products for $L e$ equal to $0.8,1.0$, and 1.2; vortex-stretching and viscous dissipation are the leading contributors, while the remaining contributions are slightly smaller although non-negligible. By contrast, the mean value of enstrophy decreases from the leading edge before increasing up to the trailing edge of the flame; in these cases, the mean value of baroclinic torque is significantly greater than the other contributions in most of the preheat and reacting regions; vortex-stretching, destruction by volumetric dilatation rates and viscous transport, and dissipation remain comparable over most of the flame. An explanation for the significant qualitative and quantitative differences in the enstrophy transport, taking place for $L e$ between 0.6 and 0.8 for the given turbulence intensity, is sought in terms of the alignments of vorticity and the gradients of density, pressure, temperature, and reaction progress variable. The transport statistics of the enstrophies of the vorticity vector components tangential and normal to iso-scalar surfaces, $c(\mathbf{x}, t)=$ constant, provide further insight into the mechanisms of the differences in the enstrophy transport in response to the changes in the global Lewis number. Published by AIP Publishing. [http://dx.doi.org/10.1063/1.4981219]
\end{abstract}

\section{INTRODUCTION}

Vorticity and enstrophy are fundamental attributes of turbulent flows ${ }^{1}$ and the investigation of their dynamics is of paramount interest. ${ }^{2}$ Therefore, vorticity and enstrophy budgets must be unmistakably related to those of kinetic energy and its dissipation rate within the flame. The longstanding issue of flame-generated turbulence in combusting flows, which has been experimentally and numerically investigated, ${ }^{3-14}$ might find sound answers by examining the vorticity dynamics. A definition of flame-generated turbulence has been proposed, determining experimentally that "in some cases the flame damps the turbulence whereas in most other instances it generates additional turbulence," estimating that the "total magnitude of the relative flame-generated turbulence intensity does not exceed about 12\%." 6 The turbulent kinetic energy budget for a premixed methane-air flame has been investigated through the measurement of the various terms, finding

a)Electronic mail: 1uis.cifuentes@uni-due.de that flame-generated turbulence increases with heat release. ${ }^{7}$ On the other hand, Favre averaged turbulent kinetic energy in premixed turbulent flames has been computed, showing that for large ratios of unburned reactants and burned products densities, the axial turbulent kinetic energy "increases by a factor of 17 through the flame, while the variances of the other two components increase by just 50\%." ${ }^{\circ}$ A direct numerical simulations (DNS) database of a turbulent premixed flame has been used to understand the mechanisms of flame-generated turbulence and assess the performance of zone-conditional two-fluid equations. $^{14}$

Several features of vorticity and its alignment with the eigenvectors of the strain rate tensor for turbulent nonpremixed $^{15-18}$ and premixed ${ }^{19-28}$ flames have been scrutinized. The important effects of the Lewis number, Le, on the geometry and statistical features of turbulent premixed flames has been unveiled using DNS $;{ }^{29,30}$ Chakraborty $^{31}$ has shown that the vorticity statistics and its alignment with the principal directions of strain are significantly affected by the values of $L e$, with profound differences for the "thin reaction zone" and the "corrugated flamelet" regimes. Chakraborty et al. ${ }^{32}$ have 
examined DNS data sets for a statistically planar turbulent premixed flames to investigate the anisotropic behavior within the flame brush due to the baroclinic torque of the average vorticity components and to reveal that the correlation between the fluctuations of enstrophy and dilatation rate plays an important role in determining the material derivative of enstrophy based on the mean flow in the case of a low $L e$.

Gorski and Bernard ${ }^{33}$ proposed an enstrophy transport equation in the context of Reynolds Averaged Navier Stokes (RANS) modeling. Bobbitt et al. ${ }^{34}$ examined DNS data sets to investigate changes in turbulence (linearly forced) characteristics across high Karlovitz $(\mathrm{Ka})$ number n-heptane/air premixed flames, modeled with a 35-species finite-rate chemical mechanism; they found vorticity to scale with the Kolmogorov time micro-scale and vortex-stretching and viscous dissipation to be the leading terms in the enstrophy transport equation. Bobbitt and Blanquart ${ }^{35}$ used the same DNS database to investigate the isotropy of the smallest turbulent scales through the analysis of the vorticity vector; vortexstretching is responsible for anisotropy, which reduces as $\mathrm{Ka}$ and the integral-scale/flame thickness ratio increases. For high $K a$, the local dynamics of vorticity and stretching resembles that of homogeneous isotropic turbulence, whereas the flame modifies vortex-stretching and vorticity alignments with the strain-rate tensor eigenvectors at low Ka. Ranjan et al. ${ }^{36}$ used three DNS data sets for freely propagating $\mathrm{CH}_{4}$-air statistically planar premixed flames interacting with a field of decaying homogeneous turbulence to test several Large Eddy Simulation (LES) modeling strategies with finite-rate chemistry; three different combinations of low, intermediate, and high $K a, R e$, and turbulence r.m.s/laminar flame-thickness, and comparable integral-scale/flame thickness ratios were explored; they obtained that the characteristic length of vorticity structures increases across the flames and that vortexstretching and "viscous dissipation" are leading terms for intermediate and high $K a$, while the baroclinic torque plays a minor role and the dilatation term can be negative for high $K a$.

The aim of the present analysis is to investigate the influence of the Lewis number on the instantaneous vorticity and enstrophy fields of a turbulent premixed statistically planar flame. An existing DNS database ${ }^{37-39}$ for statistically planar turbulent premixed flames with a simple one-step Arrheniustype chemical conversion has been examined, and the various terms in the enstrophy transport equation have been computed across the flame. There have been several previous analyses $^{29,30,40-42}$ where single-step chemistry is used to analyse the effects of the global Lewis number in isolation, and the same approach has been adopted here. The statistical behaviours of vorticity and enstrophy transport in premixed turbulent flames are principally driven by fluid-dynamics and the pressure and density gradients induced by the thermal field. These aspects are influenced by global dependences of the chemical reaction rate on reaction progress variable and temperature fields, which are satisfactorily captured by simple one-step chemistry; this is confirmed by comparing the reaction progress variable dependence of chemical reaction rate in Chakraborty and Cant ${ }^{43}$ and Chakraborty et al. ${ }^{44}$ Similarly, the statistics of scalar-gradients obtained from simple chemistry $\mathrm{DNS}^{45-47}$ are found to be qualitatively similar to the corresponding results from detailed chemistry DNS data. ${ }^{44,48,49}$

This work presents a detailed parametric DNS data analysis to scrutinize the effects of the global Lewis number on the statistical behaviour of enstrophy transport across statistically planar turbulent premixed flames. Some objectives of this research are as follows:

- To present simple vorticity and enstrophy transport equations with every term having a clear physical meaning, and also to decompose the total enstrophy into the additive contributions of the vorticity vector components normal and tangential to iso-scalar surfaces obtaining their evolution equations.

- To examine the relative importance of the various terms contributing to the total, normal, and tangential enstrophies for different Lewis numbers.

- To explain the increasing importance of the baroclinic torque as the Lewis number decreases and the influences of diffusive-thermal instability aftereffects manifest; alignments among the various vector fields contributing to the baroclinic torque in the enstrophy transport equation are scrutinized, and its relative contributions to normal and tangential enstrophies are obtained.

This work explores the influence of the global Lewis number on the flow vorticity dynamics across statistically planar turbulent premixed flames. Causes for the large values of the mean baroclinic torque conditional upon the reaction progress variable for small values of the global Lewis number are investigated; alignments among the various vector fields contributing to the baroclinic torque in the enstrophy transport equation are scrutinized. Contributions to the transport equations of enstrophies ascribed to the vorticity components normal and tangential to iso-scalar surfaces are also examined and yield further insight into the vorticity evolution within turbulent flames.

Section II concentrates on the derivation of the total enstrophy transport equation and of that of its additive constituents due to the vorticity components tangential and normal to iso-scalar surfaces. Some brief information on the numerics of the DNS code used to generate the analyzed dataset is provided in Section III. Main results are presented and discussed in Section IV. Conclusions are summarized in Section V.

\section{MATHEMATICAL DESCRIPTION}

A vorticity transport equation can be derived, starting from the flow momentum equation

$$
\frac{\partial u_{i}}{\partial t}+u_{j} \frac{\partial u_{i}}{\partial x_{j}}=-\frac{1}{\rho} \frac{\partial p}{\partial x_{i}}+\frac{1}{\rho} \frac{\partial \tau_{i j}}{\partial x_{j}}
$$

where $p$ is the pressure and the viscous stress tensor, $\tau_{i j}$, obeys the Navier-Poisson constitutive relation

$$
\tau_{i j}=2 \mu S_{i j}-\frac{2}{3} \mu S_{k k} \delta_{i j},
$$


where $\mu$ is the dynamic viscosity coefficient, taken as a constant. Here, $S_{i j}$ is the rate of strain tensor defined by

$$
S_{i j}=\frac{1}{2}\left(\frac{\partial u_{i}}{\partial x_{j}}+\frac{\partial u_{j}}{\partial x_{i}}\right) \text {. }
$$

Applying the curl operator, $\varepsilon_{i j k} \partial\left(\right.$ ) $/ \partial x_{j}$, to Equation (1), it is possible to obtain the vorticity transport equation

$$
\begin{aligned}
\frac{\partial \omega_{i}}{\partial t}+u_{j} \frac{\partial \omega_{i}}{\partial x_{j}}= & S_{i j} \omega_{j}-S_{j j} \omega_{i}+\varepsilon_{i j k} \frac{1}{\rho^{2}} \frac{\partial \rho}{\partial x_{j}} \frac{\partial p}{\partial x_{k}} \\
& -\varepsilon_{i j k} \frac{1}{\rho^{2}} \frac{\partial \rho}{\partial x_{j}} \frac{\partial \tau_{k l}}{\partial x_{l}}+v \nabla^{2} \omega_{i} .
\end{aligned}
$$

The vorticity vector is defined by $\omega_{i}=\varepsilon_{i j k} \partial u_{k} / \partial x_{j}$ and $\varepsilon_{i j k}$ is the Levi-Civita alternating tensor. The first term on the right side of Equation (4) represents the vortex-stretching, while the second one stands for the vorticity annihilation/generation by positive/negative volumetric dilatation rates. The third and fourth terms embody the torques by pressure and viscous forces, respectively. The fifth term denotes the viscous diffusion of vorticity.

Multiplying Equation (4) by $\omega_{i}$, one can readily obtain a transport equation for the enstrophy, $E=\omega_{i} \omega_{i} / 2$,

$$
\begin{aligned}
\frac{\partial E}{\partial t}+u_{j} \frac{\partial E}{\partial x_{j}}= & \underbrace{\omega_{i} S_{i j} \omega_{j}}_{T_{1}}-\underbrace{2 E S_{j j}}_{T_{2}}+\underbrace{\varepsilon_{i j k} \frac{\omega_{i}}{\rho^{2}} \frac{\partial \rho}{\partial x_{j}} \frac{\partial p}{\partial x_{k}}}_{T_{3}} \\
& -\underbrace{\varepsilon_{i j k} \frac{\omega_{i}}{\rho^{2}} \frac{\partial \rho}{\partial x_{j}} \frac{\partial \tau_{k l}}{\partial x_{l}}}_{T_{4}}+\underbrace{\frac{\mu}{\rho}\left(\nabla^{2} E\right)}_{T_{5}}-\underbrace{\frac{\mu}{\rho}\left(\frac{\partial \omega_{i}}{\partial x_{j}} \frac{\partial \omega_{i}}{\partial x_{j}}\right)}_{T_{6}} .
\end{aligned}
$$

In Equation (5), $T_{1}$ is the enstrophy generation by vortexstretching, $T_{2}$ is the enstrophy annihilation/production by the volumetric dilatation rate, $T_{3}$ and $T_{4}$ are the contributions to $E$ of pressure (baroclinic) and viscous torques, respectively, $T_{5}$ is the viscous transport of enstrophy, and $T_{6}$ its viscous dissipation rate. The various terms $T_{1}-T_{6}$ quantify not only the magnitude of the different vectors and tensors contributing to them but also of the alignment of the vectorial terms on the right side of Eq. (4) with the vorticity vector; for example, $\omega \cdot(\mathbf{S} \cdot \boldsymbol{\omega})=|\boldsymbol{\omega}| \mathbf{S} \cdot \boldsymbol{\omega} \mid \cos (\boldsymbol{\omega},(\mathbf{S} \cdot \boldsymbol{\omega}))$ depends on the magnitudes of the vorticity and vortex-stretching vectors and on their alignment, given by the cosine of the angle between them.

The local vorticity vector can be decomposed into its components normal and tangential to an iso-scalar surface $c(\mathbf{x}, t)$ $=$ constant $^{32}$

$$
\omega_{i}=\left(\omega_{j} n_{j}\right) n_{i}+\left(\delta_{i j}-n_{i} n_{j}\right) \omega_{j},
$$

where $c$ is the reaction progress variable with local unit normal vector, $\mathbf{n}$, defined by $n=-\nabla c /|\nabla c|$.

The enstrophy associated to the normal component of the vorticity is

$$
E_{N}=\frac{1}{2}\left(\omega_{j} n_{j}\right)\left(\omega_{k} n_{k}\right),
$$

whereas that due to the tangential component is

$$
E_{T}=\frac{1}{2}\left[\left(\delta_{i j}-n_{i} n_{j}\right) \omega_{j}\right]\left[\left(\delta_{i k}-n_{i} n_{k}\right) \omega_{k}\right] .
$$

Therefore, one can write the following: $E=\omega_{i} \omega_{i} / 2=E_{N}+E_{T}$.
A transport equation for the enstrophy associated to the normal component of the vorticity can be readily derived

$$
\begin{aligned}
\frac{D E_{N}}{D t}= & \underbrace{2\left(n_{i} S_{i j} \omega_{j}+V \omega_{i} \frac{\partial n_{i}}{\partial x_{N}}\right) \omega_{N}}_{T_{N 1}}-\underbrace{\left(S_{j j}+a_{N}\right)\left(\omega_{N}\right)^{2}}_{T_{N 2}} \\
& +\underbrace{n_{i} \varepsilon_{i j k} \frac{1}{\rho^{2}} \frac{\partial \rho}{\partial x_{j}} \frac{\partial p}{\partial x_{k}} \omega_{N}}_{T_{N 3}}-\underbrace{n_{i} \varepsilon_{i j k} \frac{1}{\rho^{2}} \frac{\partial \rho}{\partial x_{j}} \frac{\partial \tau_{k l}}{\partial x_{l}} \omega_{N}}_{T_{N 4}} \\
& +\underbrace{\left(n_{i} v \nabla^{2} \omega_{i}\right) \omega_{N}}_{T_{N 5}} .
\end{aligned}
$$

The first term on the right side represents the vortexstretching contribution to $E_{N}$ and the second its annihilation by volumetric dilatation. The third and fourth terms are the generation of $E_{N}$ by the baroclinic and the viscous-force torques. The last term contains both viscous transport and dissipation of $E_{N}$.

Similarly, a transport equation for $E_{T}$ can be obtained from its definition. However, subtracting Eq. (9) from Eq. (5), an alternative transport equation for $E_{T}$ is derived.

\section{DNS DATASETS EXAMINED}

A DNS database of freely propagating statistically planar turbulent premixed flames with $L e=0.34,0.6,0.8$, 1.0 , and 1.2 is examined. It was generated using a threedimensional variable-density code SENGA, ${ }^{37}$ which solved non-dimensional mass, momentum, energy, and reaction progress variable conservation equations. This database has been used in several previous analyses and provided important insights into several aspects of combustion science and modeling. 38,39

It can be stated that the unity Lewis number flames are analogous to the stoichiometric $\mathrm{CH}_{4}$-air flame, whereas the Lewis number 0.34 case is representative of a lean $\mathrm{H}_{2}$-air mixture. The Lewis number 0.6 and 0.8 flames are representatives of $\mathrm{H}_{2}$-blended $\mathrm{CH}_{4}$-air mixtures (e.g., $20 \%$ and $10 \%$, by volume $\mathrm{H}_{2}$-blended $\mathrm{CH}_{4}$-air flames with overall equivalence ratio of 0.6) and the Lewis number 1.2 case is a representative of a hydrocarbon-air mixture involving a fuel heavier than $\mathrm{CH}_{4}$ (e.g., ethylene-air mixture with equivalence ratio of 0.7). ${ }^{50-53}$ Furthermore, the range of Lewis numbers considered here is comparable to that explored by Trouvé and Poinsot. ${ }^{41}$

\section{A. Dimensionless equations}

The full set of governing equations is made dimensionless using reference characteristic variables. The non-dimensional mass, momentum, energy, and progress variable transport equations are given by ${ }^{37}$

$$
\begin{gathered}
\frac{\partial \rho^{+}}{\partial t^{+}}+\frac{\partial\left(\rho^{+} u_{i}^{+}\right)}{\partial x_{i}^{+}}=0 \\
\frac{\partial\left(\rho^{+} u_{i}^{+}\right)}{\partial t^{+}}+\frac{\partial\left(\rho^{+} u_{k}^{+} u_{i}\right)}{\partial x_{k}}=-\frac{\partial P^{+}}{\partial x_{i}^{+}}+\frac{1}{R e} \frac{\partial\left(\tau_{k i}^{+}\right)}{\partial x_{k}^{+}}
\end{gathered}
$$




$$
\begin{aligned}
\frac{\left(\rho^{+} E^{+}\right)}{\partial t^{+}}+\frac{\left(\rho^{+} u_{k}^{+} E^{+}\right)}{\partial x_{k}^{+}}= & -(\gamma-1) M a^{2} \frac{\partial\left(P^{+} u_{k}\right)}{\partial x_{k}^{+}}+\frac{1}{\operatorname{Re}}(\gamma-1) \\
& \times M a^{2} \frac{\partial\left(\tau_{k i}^{+} u_{i}^{+}\right)}{\partial x_{k}^{+}}+\frac{\tau}{\operatorname{RePr}} \frac{\partial}{\partial x_{k}}\left(\lambda \frac{\partial T^{+}}{\partial x_{k}^{+}}\right) \\
& -\frac{\tau}{\operatorname{RePr}} \frac{\partial}{\partial x_{k}^{+}}\left(\rho D \frac{\partial c}{\partial x_{k}^{+}}\right), \\
\frac{\left(\rho^{+} c\right)}{\partial t^{+}}+\frac{\left(\rho^{+} u_{k}^{+} c\right)}{\partial x_{k}^{+}}= & \dot{\omega}^{+}+\frac{1}{\operatorname{ReSc}} \frac{\partial}{\partial x_{k}^{+}}\left(\rho D \frac{\partial c}{\partial x_{k}^{+}}\right) .
\end{aligned}
$$

All non-dimensional quantities are shown with a + superscript. In Eqs. (10)-(13), Re $=\rho_{\text {ref }} u_{r e f} L_{r e f} / \mu_{r e f}$ is the nominal Reynolds number, $M a=u_{r e f} / a_{r e f}$ is the Mach number, $\gamma=C_{p} / C_{v}$ is the ratio of specific heats, $\operatorname{Pr}$ is the Prandtl number and $S c=P r L e$ is the Schmidt number, and $\rho_{r e f}, u_{r e f}$, $L_{r e f}, a_{r e f}$, and $\mu_{r e f}$ are the reference values of density, velocity, length scale, acoustic velocity, and viscosity, respectively. Here the density, viscosity, and acoustic speed of the unburned gas are taken to be $\rho_{r e f}, \mu_{r e f}$, and $a_{r e f}$, respectively, and $S_{L}$ and $10 \delta_{t h}$ are considered to be $u_{r e f}$ and $L_{r e f}$, respectively, where $\delta_{t h}=\left(T_{a d}-T_{u}\right) / \max \left|\nabla T_{i n s}\right|_{L}$ is the thermal flame thickness, with $T_{\text {ins }}$ being the instantaneous dimensional temperature; the subscript " $L$ " refers to unstrained laminar flame quantities. The internal energy $E=C_{v} T+u_{k} u_{k} / 2+H(1-c)$ (where $H$ is the heat of reaction per unit mass of reactants consumed) is normalised by $C_{p} T_{0}$ in the following manner:

$$
E=\frac{1}{\gamma}\left(1+\tau T^{+}\right)+\frac{1}{2}(\gamma-1) M a^{2} u_{k}^{+} u_{k}^{+}+\tau(1-c),
$$

where $T^{+}=\left(T-T_{u}\right) /\left(T_{a d}-T_{u}\right)$ is the non-dimensional temperature. The gas is assumed to follow the perfect gas law $P=\rho R T$, which takes the following non-dimensional form:

$$
P^{+}=\frac{1}{\gamma M a^{2}} \rho^{+}\left(1+\tau T^{+}\right)
$$

It can easily be seen from Eqs. (10)-(15) that $u^{\prime} / S L, l / \delta_{t h}$, $\tau$, and $L e=S c / P r$ are the key non-dimensional parameters which govern the system of equations, and explicit values of $S_{L}, T_{a d}, T_{u}$, and $T_{a c}$ are not necessary for the purpose of this analysis.

\section{B. Numerical simulation}

A planar laminar flame, used as initial condition for the scalar fields, is allowed to interact with the turbulent flow, which is initialized using a precomputed incompressible homogeneous isotropic velocity field. ${ }^{54}$ The domain is considered to be periodic in the $y$ and $z$ directions, while partially non-reflecting boundary conditions are imposed in the $x$-direction, using the Navier-Stokes Characteristic Boundary Conditions (NSCBC) approach. ${ }^{54}$ In this configuration, no forcing is used to maintain the turbulence level and the flameturbulence interaction takes place under decaying turbulence, similar to previous analyses. ${ }^{29,30,40-42}$

Spatial derivatives for the internal grid points are evaluated using a tenth-order central difference scheme, with the order of differentiation dropping gradually to a one-sided second-order scheme at the non-periodic boundaries. A third order explicit Runge-Kutta scheme ${ }^{55}$ is used for time advancement.
The chemical mechanism is simplified by using a simple one-step Arrhenius-type chemical conversion for the purpose of computational economy and to explore the effects of the Lewis number, $L e$, in isolation following several previous analyses..$^{29,30,42}$ The enstrophy transport is primarily a fluiddynamic phenomenon, and the combustion process affects the enstrophy transport only through the heat release pattern. In principle, the effects of detailed chemical kinetics are relatively irrelevant because simple chemistry qualitatively captures the heat release pattern within premixed flames.

For the current study, the heat release parameter, $\tau$ $=\left(T_{a d}-T_{u}\right) / T_{u}$, and the Zel'dovich number, $\beta=T_{a c}\left(T_{a d}\right.$ $\left.-T_{u}\right) / T_{a d}^{2}$, are set equal to 4.5 and 6.0 , respectively; the subscripts " $u$ " and "b" stand for variables in "unburned" and "burnt" gases. $T_{a d}, T_{u}$, and $T_{a c}$ are the adiabatic flame, the unburned gas, and the activation temperatures, respectively. For the present analysis, the reaction progress variable, $c$, is defined based on a suitable reactant mass fraction $Y_{R}$ as follows:

$$
c=\frac{Y_{R_{u}}-Y_{R}}{Y_{R_{u}}-Y_{R_{b}}} .
$$

The reaction progress variable vanishes in the fresh reactants and monotonically grows to become unity in the hot products. $c(\mathbf{x}, t)$ obeys the conventional convection-diffusionreaction equation

$$
\frac{\partial c}{\partial t}+u_{j} \frac{\partial c}{\partial x_{j}}=\frac{1}{\rho} \frac{\partial}{\partial x_{j}}\left(\rho D \frac{\partial c}{\partial x_{j}}\right)+\dot{\omega}_{c}
$$

The computational domain is taken to be a cube with every side equal to $24.1 \delta_{t h}$, which is discretised using a uniform grid of $230 \times 230 \times 230$. It is possible to define an alternative flame thickness as $\delta_{L}=1 / \max |\nabla c|_{L}$. The thicknesses $\delta_{L}$ and $\delta_{t h}$ are not equal to each other and $\delta_{L}>\delta_{t h}\left(\delta_{L}<\delta_{t h}\right)$ for $L e<1$ ( $L e>1$ ) flames. For the $L e \approx 1.0$ (e.g., $0.8,1.0$, and $1.2), \delta_{L}$ and $\delta_{t h}$ remain comparable (i.e., $\delta_{L} / \delta_{t h} \approx 0.9,1.0$, and 1.15 for $L e=1.2,1.0$, and 0.8 ), whereas $\delta_{L} / \delta_{t h}$ is 1.4 and 2.1 for $L e=0.6$ and 0.34 flames. Thus, in all cases, either about 10 or more than 10 grid points within $\delta_{L}$ are taken. For detailed chemistry DNS, one has to resolve the reaction layer for intermediate species and thus typically 15 points are kept within the flame thickness, but for simple chemistry DNS, 10 grid points often suffices. ${ }^{56}$ The flame resolution used in this paper is consistent with existing studies, ${ }^{29,30,40-42}$ which concentrated on the effects of the global Lewis number.

The initial r.m.s. velocity to laminar burning velocity and integral length scale to flame thickness ratios of every simulation are taken to be $u^{\prime} / \mathrm{S}_{\mathrm{L}}=7.5$ and $l / \delta_{t h}=2.45$, respectively, which yield a Damköhler number $D a=\left(l / \delta_{t h}\right) /\left(u^{\prime} / S_{L}\right)=0.32$ and a Karlovitz number $K a=\left(u^{\prime} / S_{L}\right)^{3 / 2}\left(l / \delta_{t h}\right)^{-1 / 2}=13.12$.

The domain size in the canonical configuration is governed by the necessity of enough number of eddies within the computational domain. For the cases considered here, the initial value of $l / \delta_{t h}$ is taken to be 2.45 so a domain length of $24.1 \delta_{t h}$ accommodates 10 integral eddies within every side of the domain. The minimum distance of the $c=0.99$ iso-surface from the outlet boundary is of the order of $4.0 \delta_{t h}$ (i.e., greater than or equal to 40 grid points). Reducing the domain size by $1.5 \delta_{t h}$ on every side had a marginal effect on the statistics (e.g., maximum difference in $|\nabla c|$ values was about $0.5 \%$ ). 
The grid spacing $\Delta x$ for a $230 \times 230 \times 230$ grid amounts to $\eta=1.4 \Delta x$, and making the grid coarser to $200 \times 200 \times 200$ (which amounts to $\eta=1.2 \Delta x$ ) did not change the statistical behaviour (e.g., maximum difference in $|\nabla c|$ values was about $0.1 \%$ ). The domain size remains either comparable or greater than that used in published works. ${ }^{29,30,40-42}$

In decaying turbulence simulations, the desirable simulation time $t_{\text {sim }}$ is given by $t_{\text {sim }} \geq \max \left(t_{f}, t_{c}\right)$ (where $t_{f}=l / u^{\prime}$ is the initial eddy turnover time and $t_{c}=\delta_{t h} / S_{L}$ is the chemical time scale), which guarantees that the statistics can be extracted at a time when the flow field is in principle independent of initial conditions. Here, the simulation time is equal to one chemical time, which corresponds to 3.34 initial integral eddy turnover times. This simulation time is comparable to that in several previous analyses, which contributed to the fundamental understanding of turbulent premixed combustion. . $^{29,30,42,57}$ The averaging done here is ensemble averaging on a given $c$-isosurface at the time when the statistics were extracted and the same approach was adopted in several previous analyses. ${ }^{45,57}$ All the statistical behaviours shown in the paper remain qualitatively unaltered since halfway through the simulation. The temporal evolution of turbulent flame speed for the cases considered here is provided in Fig. 2(e) of Chakraborty and Cant, ${ }^{39}$ which is not repeated in this paper for the sake of brevity. The temporal evolution of flame reveals that the turbulent flame speed was not changing rapidly with time for the $L e=0.8,1.0$, and 1.2 cases. The effects of the thermodiffusive instability strengthen with decreasing Lewis number. Thus, a quasi-stationary state is not obtained for the $L e=0.34$ and 0.6 cases considered here, and perhaps it is not physically sound to expect a quasi-stationary state for all cases. A similar behaviour was reported by Trouvé and Poinsot. ${ }^{41}$

The root-mean-square velocity fluctuation in the unburned gas ahead of the flame (evaluated using the samples corresponding to $c<0.01$ ) decayed by about $50 \%$, whereas the integral length scale increased by a factor of 1.7 in comparison to its initial value. The Zel'dovich flame thickness $\alpha_{T_{0}} / S_{L}$ (where $\alpha_{T_{0}}$ is the thermal diffusivity in the unburned gas) scales with $\delta_{t h}$ (here $\delta_{t h} \approx 2 \alpha_{T_{0}} / S_{L}$ ), and, thus, the combustion takes place in the "thickened-wrinkled flame" regime for the values of $u^{\prime} / S L$ and $l / \delta_{t h}$ considered here. The scaling arguments for the regime diagram are strictly valid for the unity Lewis number, and, therefore, the non-unity Lewis number flames are nominally within the thin reaction zone regime. It has been demonstrated by Chakraborty and $\mathrm{Cant}^{39}$ that the magnitude of flame wrinkling in this analysis is at least representative for some laboratory flames. ${ }^{40}$ Further information regarding this database can be found elsewhere. ${ }^{9,31,38,57}$

\section{RESULTS AND DISCUSSION}

The variation of the mean value of $\dot{\omega}_{c}$ conditional on $c$ for the turbulent flames considered here is shown in Figure 1; illustratively, a preheat region, $0.3<c<0.75$, and a burning zone, $0.75<c<0.95$, can be delimited, although they are not essential for the results and conclusions of this work. The reactants diffuse faster into the reaction zone than the rate of thermal diffusion for $L e<1$ flames, which gives rise to higher rate of burning than the corresponding $L e=1$ flames due to

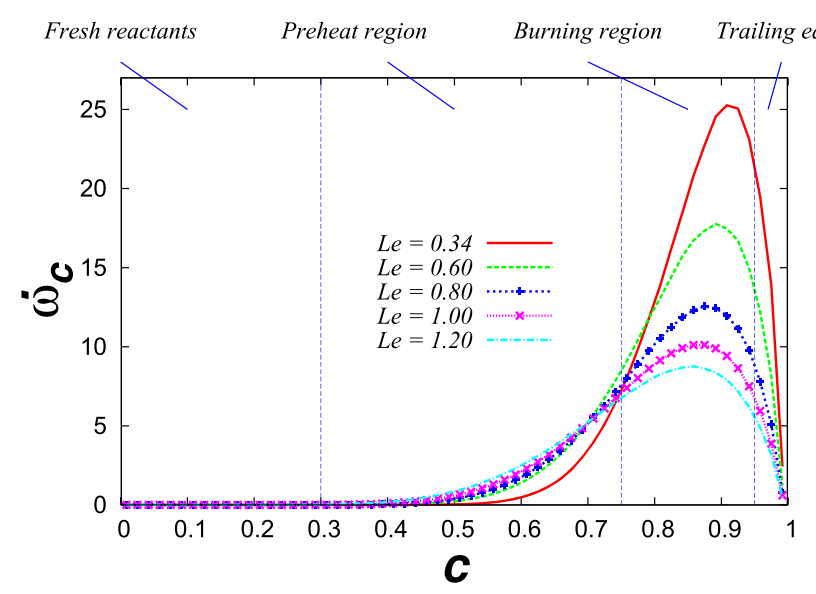

FIG. 1. Variation of mean values of reaction rate, $\dot{\omega}_{c}=\dot{\omega} / \rho$, conditional upon $c$, for different Lewis numbers. Dotted lines mark the limits of the four regions of the computational domain.

simultaneous presence of high temperature and high reactant concentration in the reaction zone. By contrast, thermal diffusion is faster than the reactant diffusion in the $L e>1$ flames, which leads to a reduction in the rate of burning due to relatively lower temperature and reactant concentration within the reaction zone in these flames than in the corresponding $L e=1$ flame. The aforementioned physical mechanisms are responsible for the increasing trend of $\dot{\omega}_{c}$ with decreasing Le. Table I lists the normalised values of the volume-integrated reaction rate of progress variable $R_{T} / R_{L}$ (where $R=\int_{V} \rho \dot{\omega}_{c} d V$ and the subscripts $T$ and $L$ are used for turbulent and laminar flame conditions, respectively), which show that $R_{T} / R_{L}$ increases significantly with decreasing $L e$, consistent with the above discussion. Interested readers are referred to Refs. 9, 29-31, 38 , and 57 and references therein for further discussion on the augmentation of burning rate and flame surface area with decreasing global Lewis number.

Muppala et al. ${ }^{50}$ experimentally obtained that $\left(A_{T} / A_{L}\right.$ - 1) scales with $(1 / L e)\left(u^{\prime} / S_{L}\right)^{0.3} R e_{t}^{0.25}$. For the values of $u^{\prime} / S_{L}$ and $l / \delta_{t h}$ at the time when statistics were extracted (presented in Table 2 in Chakraborty and $\mathrm{Cant}^{58}$ ), the value of $\left[\left(A_{T} / A_{L}-1\right) L e /\left(u^{\prime} / S_{L}\right)^{0.3} R e_{t}^{0.25}\right] /\left[\left(A_{T} / A_{L}-1\right) L e /\left(u^{\prime} / S_{L}\right)^{0.3}\right.$ $\left.R e_{t}^{0.25}\right]_{(L e=1)}$ remains of order unity (i.e., 1.05, 1.06, 1.02, and 1.03 for the $L e=0.34,0.6,0.8$, and 1.2 flames, respectively) for all the non-unity Lewis number cases, which is in good agreement with the findings of Muppala et al. ${ }^{50}$

Figure 2 presents an instantaneous snapshot of enstrophy $E=\omega_{i} \omega_{i} / 2$ contours in a plane, midway of the $y$ coordinate of

TABLE I. The effects of the Lewis number on the normalised volumeintegrated reaction rate of progress variable $R_{T} / R_{L}$ after 3.34 initial eddy turnover times.

\begin{tabular}{lc}
\hline \hline Le & $R_{T} / R_{L}$ \\
\hline 0.34 & 13.70 \\
0.60 & 4.58 \\
0.80 & 2.53 \\
1.00 & 1.83 \\
1.20 & 1.50 \\
\hline
\end{tabular}




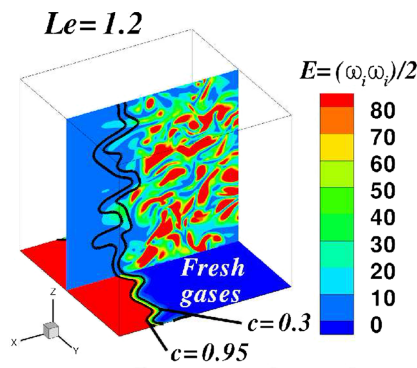

$$
L e=0.6
$$

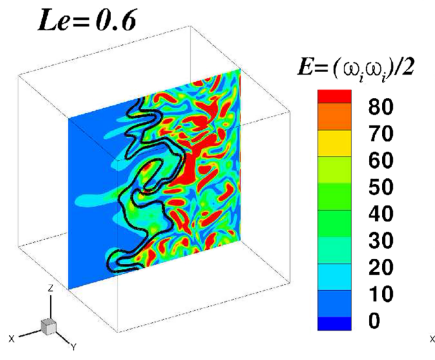

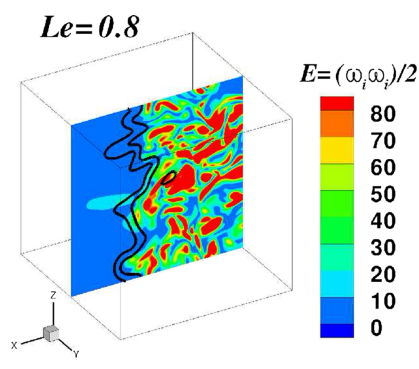

$L e=0.34$

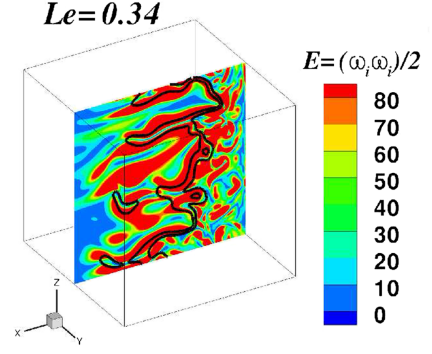

FIG. 2. Instantaneous enstrophy, $E=$ $\left(\omega_{i} \omega_{i}\right) / 2$, at different Lewis numbers. $E$ has been normalized with $\left(\delta_{t h} / S_{L}\right)^{2}$ corresponding to the $L e=1.0$ flame. Isolines of the progress variable $c=0.3$ and $c=0.95$ mark the limits for the preheat and burning regions. the computational domain, at time $t=\delta_{t h} / S_{L}$ when the statistics were extracted. This instantaneous enstrophy distribution helps to visualize its generation across turbulent premixed flames due to the variation in $L e$. It is apparent that a major part of the flame acts as an enstrophy suppressor for the cases with $L e \geq 0.80$. On the contrary, the flame apparently augments the enstrophy of the flow downstream of the flame for the $L e=0.34$ and 0.6 cases. However, the extent of enstrophy augmentation in the $L e=0.6$ case is much weaker than in the $L e=0.34$ flame .

The above observations could be substantiated further from the variations of mean enstrophy, conditioned upon the reaction progress variable, $c$, as shown in Figure 3. A slight increment of enstrophy for $L e=0.60$ can be observed within the flame, in the progress variable range $0.3 \leq c \leq 0.95$, but this augmentation of $E$ is particularly pronounced for the $L e=0.34$ case.

To understand the causes of the previous behavior, the mean values of the different terms in the enstrophy transport equation, conditional on the value of $c$, for different Lewis numbers are shown in Figure 4. At a given instant, these terms

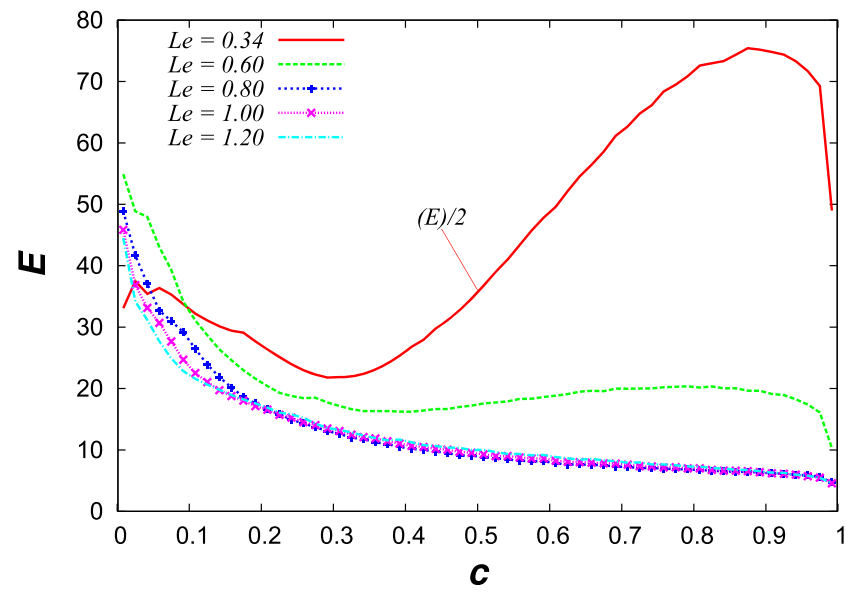

FIG. 3. Mean enstrophy, $E=\left(\omega_{i} \omega_{i}\right) / 2$, conditional upon $c$, at different $L e$. The enstrophy $E$ has been normalized with $\left(\delta_{t h} / S_{L}\right)^{2}$ corresponding to the $L e=1.0$ flame. The mean value of enstrophy for $L e=0.34$ has been divided by two. do not necessarily balance and the imbalances of contributions to the right side of Eq. (5) (i.e., the left side or material derivative of the enstrophy of a fluid particle) are plotted for various cases. An overall destruction of enstrophy is apparent across the flame for $L e$ equal to $0.8,1.0$, and 1.2, while there is a moderate (high) generation of enstrophy within the flame for $L e=0.6(L e=0.34)$.

For the $L e=0.8,1.0$, and 1.2 cases, the different terms display similar trends; vortex-stretching and viscous dissipation are the dominant mechanisms towards the unburned gas side of the flame front. Within the flame, all terms display a comparable contribution to enstrophy generation. Moreover, the mean value of viscous transport of enstrophy conditional on $c$ in these cases is not negligible compared to the mean viscous dissipation. On the other hand, annihilation/generation by volumetric dilatation rates is negative as a consequence of heat release by the flame, nearly vanishes at the extreme values of $c$, and becomes more important as the Lewis number decreases due to a greater extent of burning with decreasing $L e$. The baroclinic torque exhibits a positive contribution to the enstrophy transport over most values of $c$, with a maximum within the preheat zone, almost vanishing at $c=0$ and $c$ $=1$ and slightly increasing as $L e$ decreases. The viscous torque has a small, though not negligible, contribution for the cases considered here.

It can also be seen in Figure 4 that the baroclinic torque plays a particularly important role for the $L e=0.34$ and $L e=0.60$ cases and exhibits very large maxima near the most reactive region within the flame; small negative values towards the unburned gas give way to large positive values for the major part of the rest of the flame front. Vortex-stretching and viscous dissipation for $L e=0.6$ show similar trends to those for the cases with $L e \geq 0.8$ and assume comparable values; for $L e$ $=0.34$, vortex-stretching is approximately constant for all values of $c$, whereas viscous dissipation takes small values in the fresh reactants, which increase within the flame due, among other causes, to the increment of the kinematic viscosity. The behavior of the viscous diffusive transport of enstrophy for $L e=0.34$ and $L e=0.6$ is rather different from that at larger Lewis numbers; it changes within the flame from positive 
$L e=1.2$

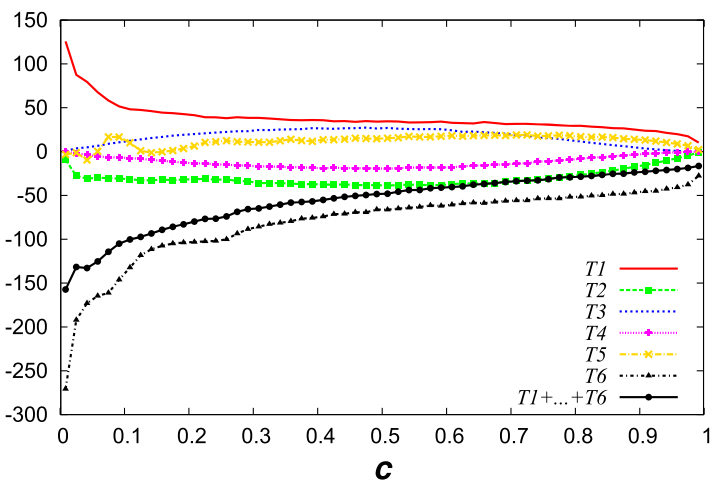

(a)

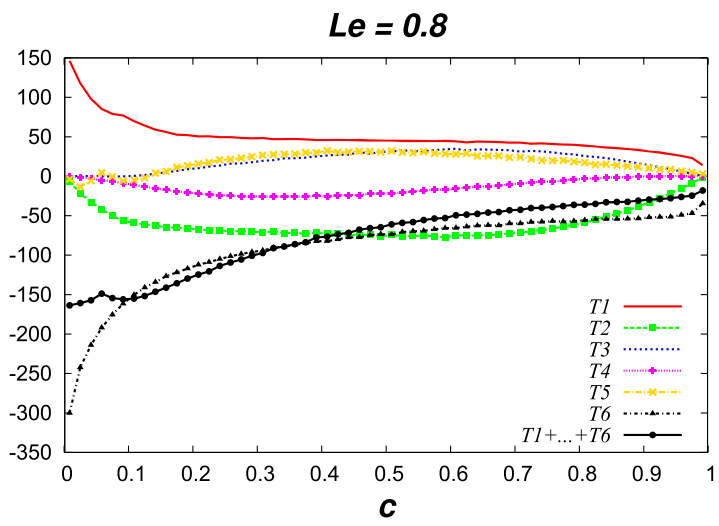

(c)
$L e=1.0$

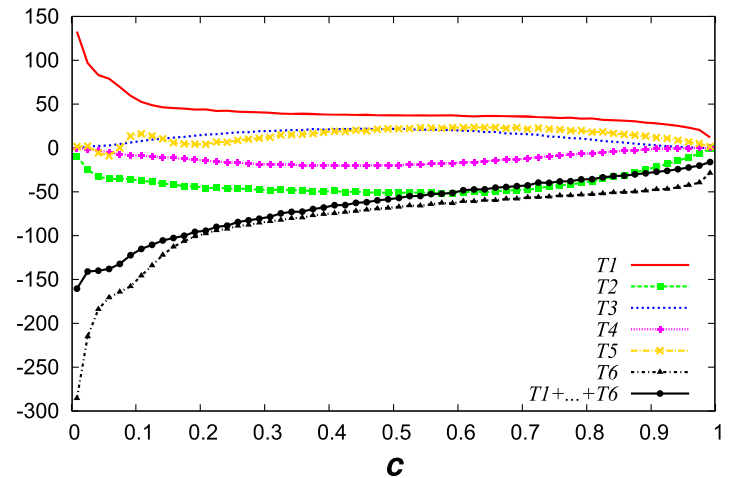

(b)

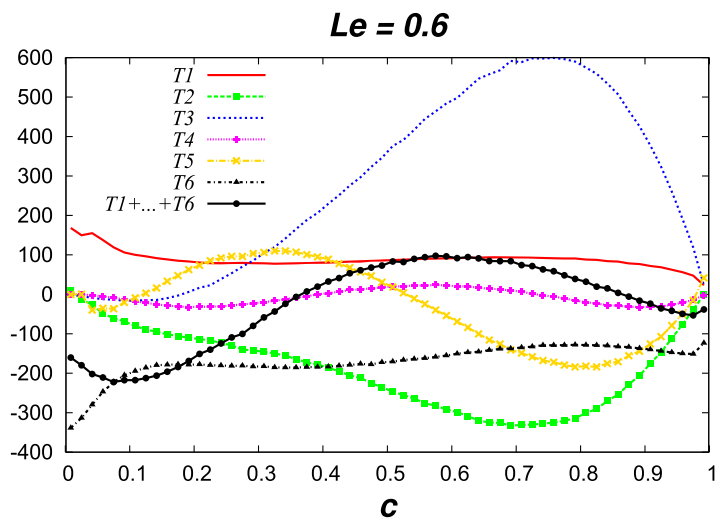

(d)

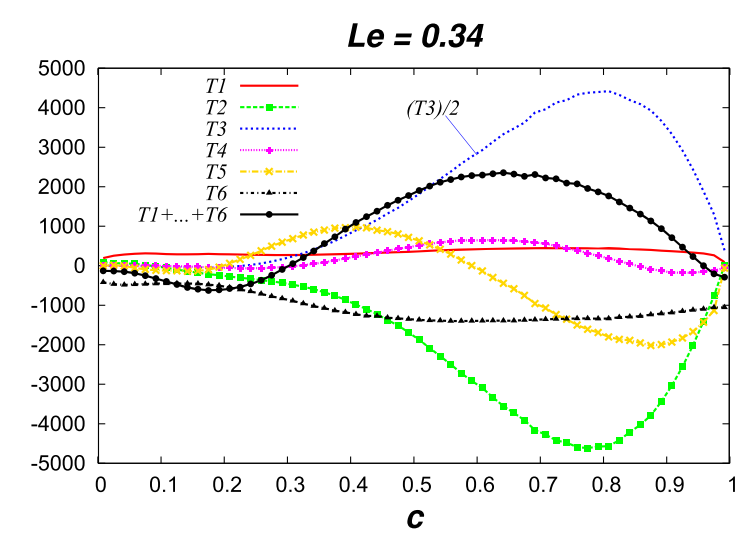

(e)

FIG. 4. Mean values of the various terms in the enstrophy conservation Equation (9), conditional upon $c$. (a) $L e=1.2$, (b) $L e=1.0$, (c) $L e=0.8$, (d) $L e=0.6$, and (e) $L e=0.34$. Variables have been normalized with $\left(\delta_{t h} / S_{L}\right)^{3}$ corresponding to the $L e=1.0$ flame. The mean value of $T_{3}$ for $L e=0.34$ has been divided by two.

values to negative ones around the middle of the flame, and the magnitude of mean viscous transport becomes greater than mean viscous dissipation around the maximum values of the chemical conversion rate. The mean viscous torque contribution is smaller than the remaining terms throughout all values of $c$ for $L e=0.6$, but it becomes comparable to the mean contribution of the vortex-stretching term for $L e=0.34$. Enstrophy annihilation by volumetric dilatation rates, due to a large heat release rate, becomes a leading sink and dominates over the viscous dissipation for a significant portion of the composition domain $(c>0.4)$. The enstrophy generation induced by the baroclinic torque is partially countered by the remaining five terms.
The magnitudes of the vorticity, $\omega$, and the vortexstretching, $\mathbf{S} \cdot \boldsymbol{\omega}$, and the cosine of the angle of these two vectors are shown in Figure 5. The alignment vorticity/vortexstretching lessens as flames become more convoluted for decreasing Lewis numbers. The variations of the three magnitudes across the flame explain the patterns of $T_{1}$ depicted in Figure 4 for different values of $L e$. The positive volumetric dilatation rate due to heat release, shown in Figure 6, implies that term $T_{2}$ is a sink of enstrophy over all values of $c$, consistent with features of Figure 4; the annihilation rate is more pronounced for lower Lewis numbers, due to more intense heat release rates. The variation of $|\omega|$ is consistent with the variation of $E$ shown in Figure 3. The augmentation of burning rate 


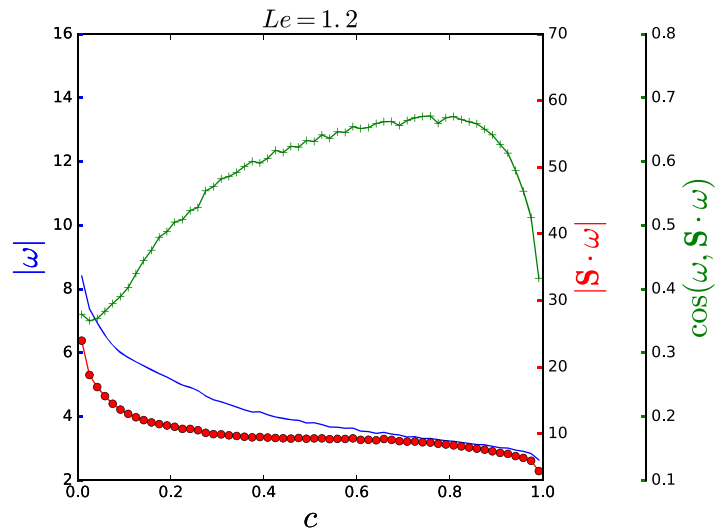

(a)

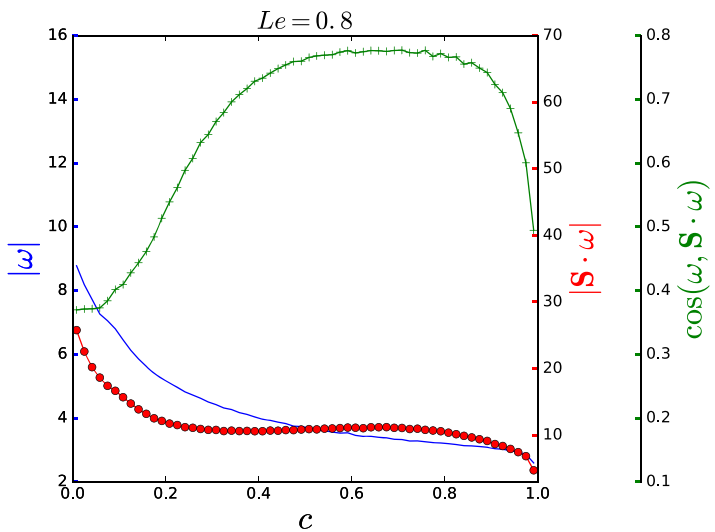

(c)

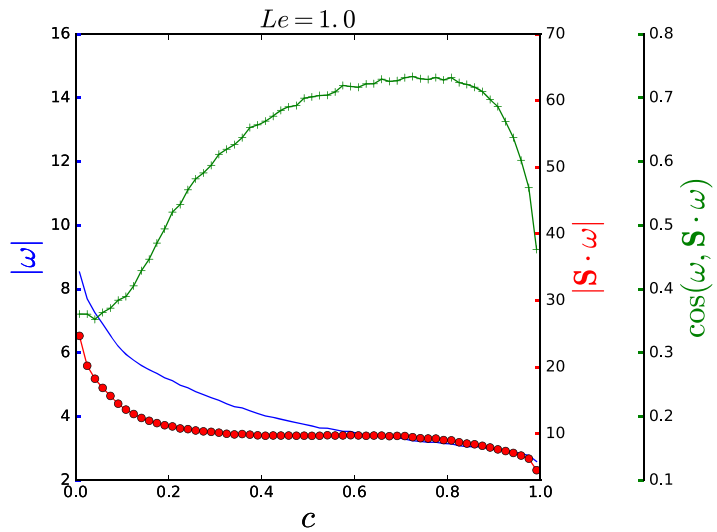

(b)

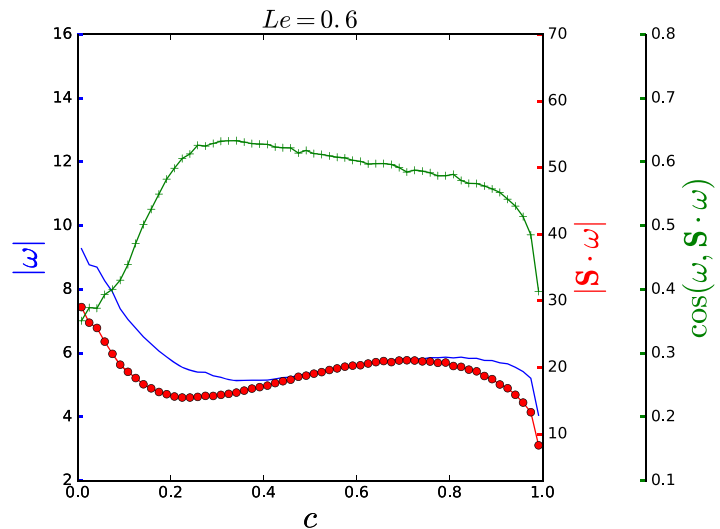

(d)

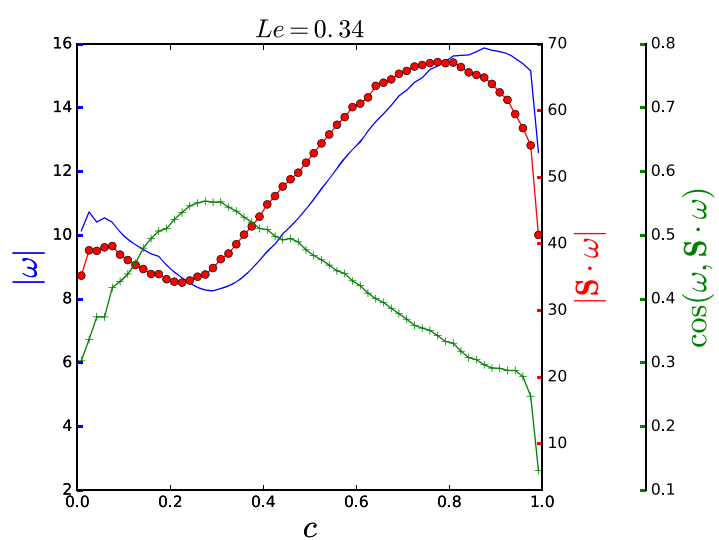

(e)

FIG. 5. Mean values of vorticity, $|\omega|$, and vortex-stretching, $|\mathbf{S} \cdot \omega|$, magnitudes and of $\cos (\omega, \mathbf{S} \cdot \boldsymbol{\omega})$, conditional upon $c$. (a) $L e=1.2$, (b) $L e=1.0$, (c) $L e=0.8$, (d) $L e=0.6$, and (e) $L e=0.34 . \omega$ and $(S \cdot \omega)$ have been normalized with $\left(\delta_{t h} / S_{L}\right)$ and $\left(\delta_{t h} / S_{L}\right)^{2}$, respectively, corresponding to the $L e=1.0$ flame.

with decreasing $L e$ gives rise to stronger thermal expansion effects for flames with smaller value of $L e$. This can be substantiated from the mean values of $S_{i j}=\nabla \cdot \mathbf{u}$ conditional on $c$ shown in Fig. 6, which reveals an increase in the magnitude of $S_{j j}$ with decreasing $L e$. Strong flame normal acceleration and high extent of flame wrinkling contribute to an increase in the magnitude of strain rates with decreasing $L e$. This is reflected in the increases in the magnitude of $|\mathbf{S} \cdot \omega|$ with decreasing Lewis number $L e$. The vortex-stretching term $T_{1}$ can be rewritten as $T_{1}=\omega \cdot(\mathbf{S} \cdot \boldsymbol{\omega})=|\omega||\mathbf{S} \cdot \boldsymbol{\omega}| \cos (\boldsymbol{\omega},(\mathbf{S} \cdot \boldsymbol{\omega}))$ $=2\left(\mathbf{e}_{\alpha} \cos ^{2} \alpha+\mathbf{e}_{\beta} \cos ^{2} \beta+\mathbf{e}_{\gamma} \cos ^{2} \gamma\right) E$, where $\mathbf{e}_{\alpha}, \mathbf{e}_{\beta}$, and $\mathbf{e}_{\gamma}$ are the most extensive, intermediate, and the most compressive principal strain rates, respectively, and the angles between $\omega$ and the eigenvectors associated with $\mathbf{e}_{\alpha}, \mathbf{e}_{\beta}$, and $\mathbf{e}_{\gamma}$ are given by $\alpha, \beta$ and $\gamma$, respectively. It has been shown by Chakraborty ${ }^{31}$ that $\omega$ predominantly aligns with $\mathbf{e}_{\beta}$ (i.e., $|\cos \beta| \approx 1$ ) but the extent of alignment with $\mathbf{e}_{\alpha}$ increases in the regions of high chemical heat release for $L e \approx 1.0$ cases. The extent of $\omega$ alignment with $\mathbf{e}_{\alpha}\left(\mathbf{e}_{\gamma}\right)$ decreases (increases) with decreasing Lewis number ${ }^{31}$ and interested readers are referred to Ref. 31 for relevant explanations. The probability of increased alignment with the most compressive (i.e., most negative) principal 


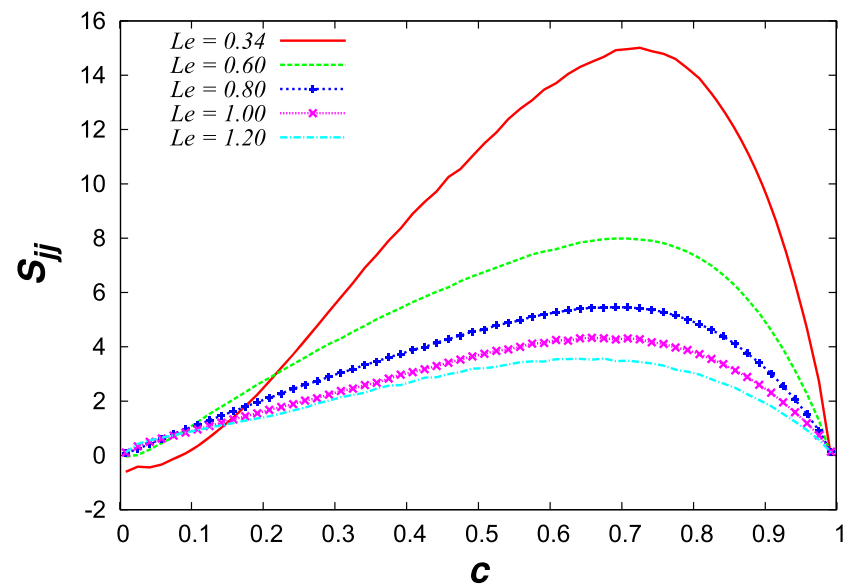

FIG. 6. Volumetric dilatation rate, $S_{j j}$, conditional upon $c$, at different $L e . S_{j j}$ has been normalized with $\left(\delta_{t h} / S_{L}\right)$ corresponding to the $L e=1.0$ flame.

strain rate is responsible for the reduction of the mean value of $\cos (\omega,(\mathbf{S} \cdot \omega))$ with decreasing $L e$.

The term $T_{3}$, the baroclinic torque contribution to $E$ in Equation (5), can be expressed as follows:

$$
\begin{aligned}
\frac{1}{\rho^{2}} \omega \cdot[(\nabla \rho) \times(\nabla p)]= & \frac{1}{\rho^{2}}|\omega||(\nabla \rho) \times(\nabla p)| \cos (\omega, \nabla \rho \times \nabla p) \\
= & \frac{1}{\rho^{2}}|\omega||\nabla \rho||\nabla p||\sin (\nabla \rho, \nabla p)| \\
& \times \cos (\omega, \nabla \rho \times \nabla p)
\end{aligned}
$$

The sizable growth of enstrophy with the reaction progress variable for the $L e=0.34$ and 0.6 cases can be explained in terms of the variations of $|\omega|,|\nabla \rho|$, and $|\nabla p|$ and the corresponding alignments given by $\cos (\omega, \nabla \rho \times \nabla p)$ and $|\sin (\nabla \rho, \nabla p)|$. The sign of $\cos (\omega, \nabla \rho \times \nabla p)$ determines whether $T_{3}$ is a source or a sink of $E$. Figure 7 depicts the mean value of $\cos (\omega, \nabla \rho \times \nabla p)$ as a function of $c$ for different Lewis numbers. Enstrophy is generated within the flame $(0.30<c<0.95)$ by the baroclinic torque for all cases considered here. Some destruction of enstrophy can only occur in part of the fresh reactants for the $L e=0.34,0.6$, and 0.8 cases and in the hot products for the $L e=1.0$ and 1.2 cases. The values of $\cos (\omega, \nabla \rho \times \nabla p)$ are relatively small (less than 0.3 ) for the $L e=0.8,1.0$, and 1.2 cases and significantly higher (between

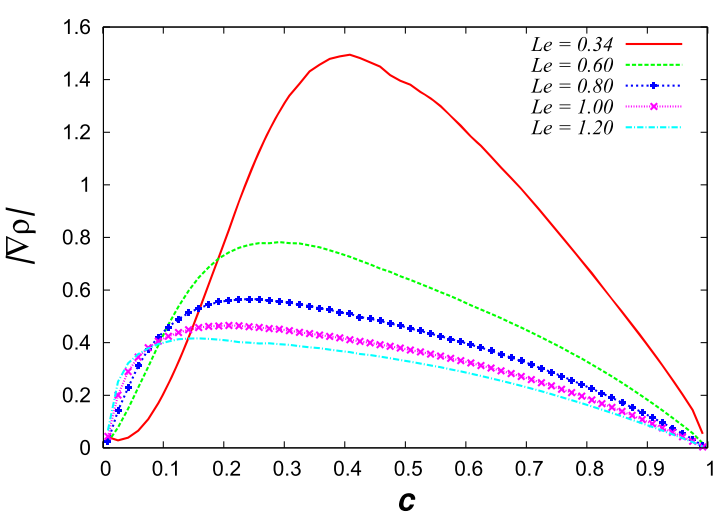

(a)

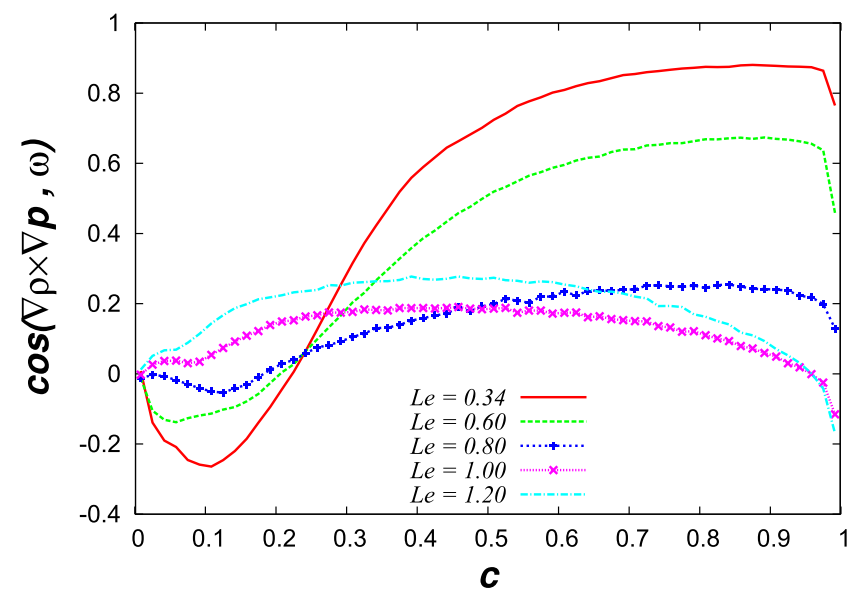

FIG. 7. Mean value of $\cos (\nabla \rho \times \nabla p, \omega)$ conditional upon $c$ for different Lewis numbers.

0.3 and 0.9 ) for the $L e=0.34$ and 0.6 cases. Therefore, the alignment of $\omega$ and $[(\nabla \rho) \times(\nabla p)] / \rho^{2}$ changes appreciably with $L e$.

The variation of the mean value of $|\omega|$ with $c$ is similar to that in Figure 3 for the enstrophy. Figure 8 shows the mean values of $|\nabla \rho|$ and $|\nabla p|$, conditional upon $c$ for different Lewis numbers. The values of $|\nabla \rho|$ and $|\nabla p|$ within the flame for $L e=0.34$ and $L e=0.6$ are considerably higher than those for the $L e=0.8,1.0$, and 1.2 cases.

Weakening of thermal diffusion rate with decreasing $L e$ increases the probability of finding high values of $\left|\nabla T_{\text {ins }}\right|,{ }^{38,45}$ which gives rise to an increase of the mean values of $|\nabla \rho|=\rho^{2} \tau\left|\nabla T_{i n s}\right| /\left[\rho_{u}\left(T_{a d}-T_{u}\right)\right]$ with decreasing Le. Furthermore, higher rate of burning for smaller values of $L e$ gives rise to stronger flame normal acceleration. This strengthening of flame normal acceleration with decreasing $L e$ is reflected in the increasing trend of mean values of $|\nabla p|$ with a decrease in the global Lewis number. Interested readers are referred to Refs. 45 and 58 for more detailed discussion on the effects of Le on both scalar and pressure gradients.

Figure 9 depicts the mean values of $|\sin (\nabla \rho, \nabla p)|$, conditional upon $c$ for different Lewis numbers; these values, particularly in the burning region, for $L e=0.34$ and $L e=0.6$, are considerably higher than the corresponding ones for the $L e$ $=0.8,1.0$, and 1.2 cases.

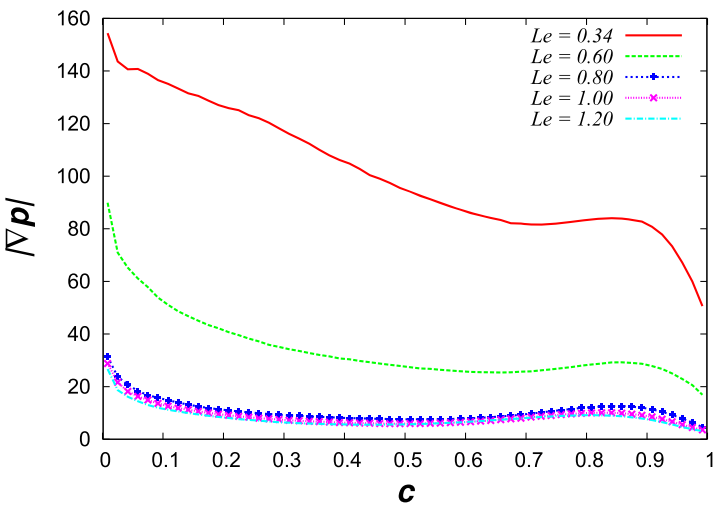

(b)

FIG. 8. Mean modulus of the (a) density gradient, $|\nabla \rho|$, and (b) pressure gradient, $|\nabla p|$, conditional upon $c$ for different Lewis numbers. $|\nabla \rho|$ and $|\nabla p|$ have been normalized by $\left(\delta_{t h} / \rho_{u}\right)$ and $\left[\delta_{t h} /\left(\rho_{u} S_{L}^{2}\right)\right]$ of the unity Lewis number flame, respectively. 


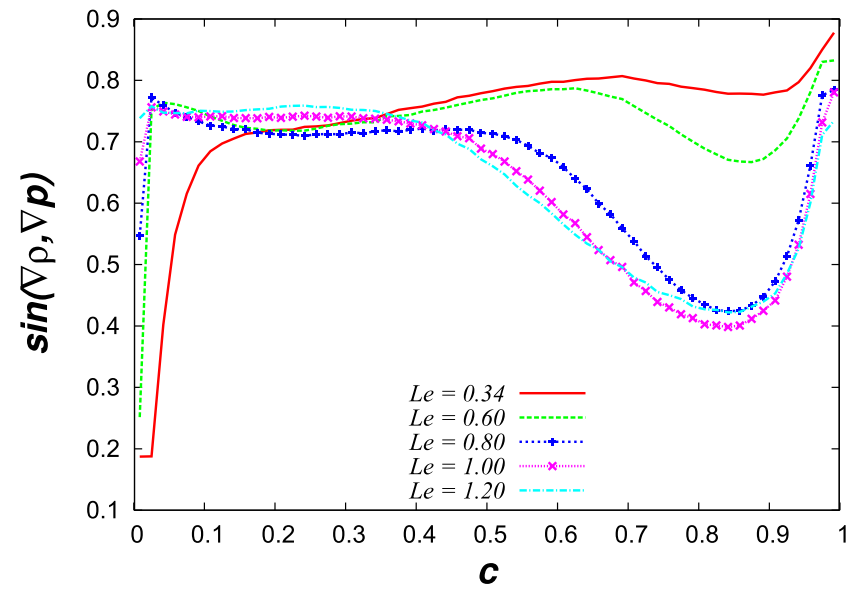

FIG. 9. Mean value of $|\sin (\nabla \rho, \nabla p)|$ conditional upon $c$ for different Lewis numbers.

Magnitudes of $|\omega|, \quad|\nabla \rho|,|\nabla p|,|\sin (\nabla \rho, \nabla p)|$, and $|\cos (\nabla \rho \times \nabla p, \omega)|$, for $L e<0.8$ cases and $c>0.3$, are considerably higher than those for $L e \geq 0.8$. This implies that an increase of the baroclinic torque contribution to $E$ in the regions with high chemical activities and this trend is particularly strong for low Lewis numbers. The latter is apparent in Figure 10 for the mean value of $T_{3}=\omega \cdot[(\nabla \rho) \times(\nabla p)] / \rho^{2}$ for various Lewis numbers; $T_{3}$ increases considerably from the preheat region to hot products for $L e=0.34$ and, to a lesser extent, for $L e=0.60$. It has been already pointed out that $T_{3}$ becomes the most important contribution to $E$ for

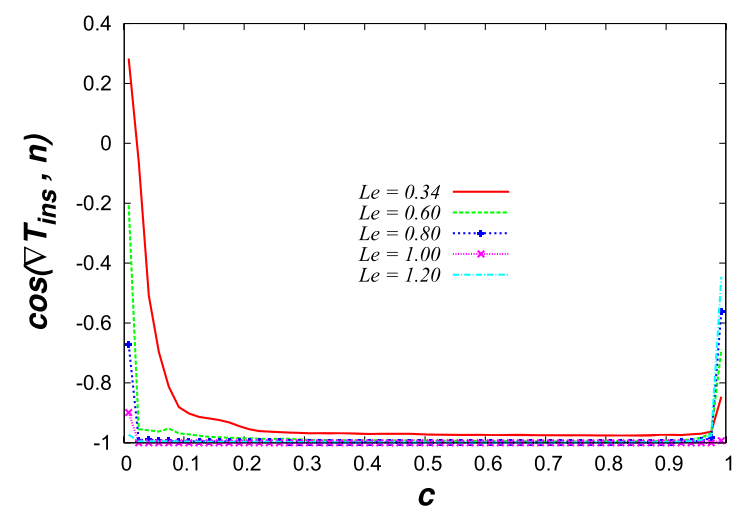

(a)

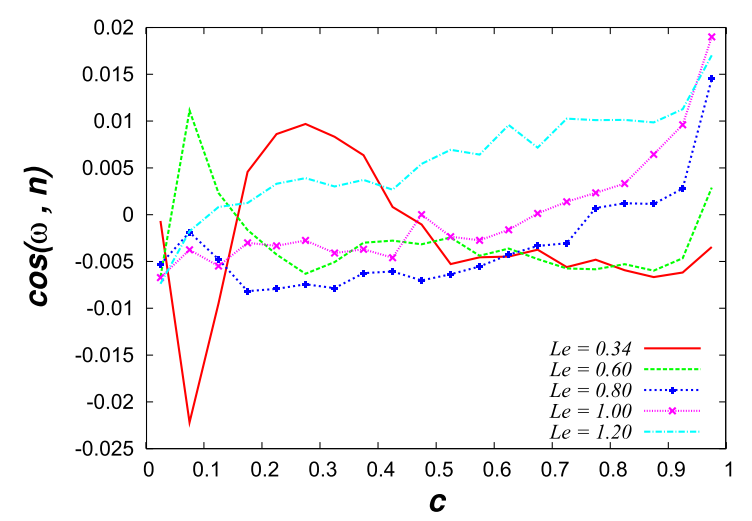

(c)

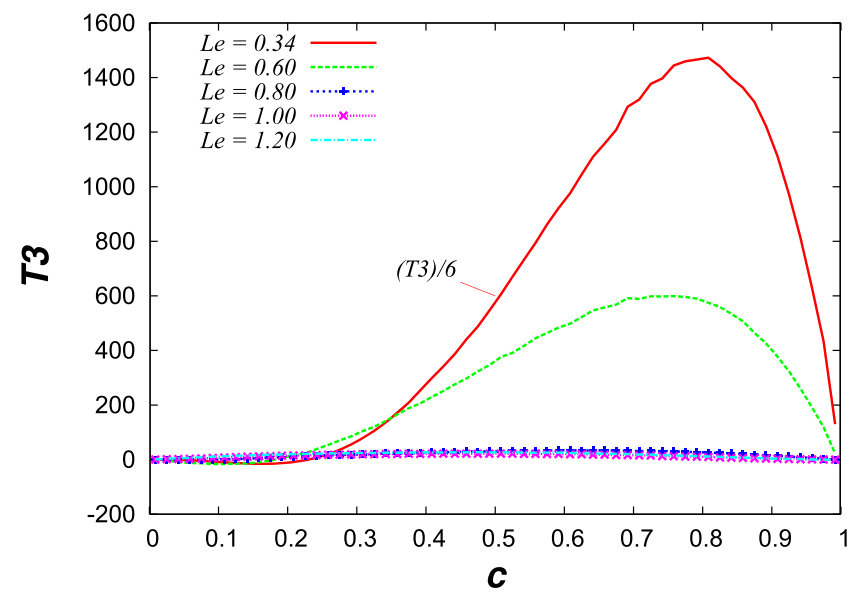

FIG. 10. Mean baroclinic torque contribution to enstrophy generation, $T_{3}$, conditional upon $c$, for different Lewis numbers. $T_{3}$ has been normalized by $\left(\delta_{t h} / S_{L}\right)^{3}$ of the unity Lewis number flame. The mean value of $T_{3}$ for $L e=0.34$ has been divided by six.

low Lewis number cases (see Figure 4). Further characterization and additional details to the previous description are provided in Figure 11, which depicts the alignments of vectors $\omega, \nabla \rho, \nabla p$, and $\nabla T_{\text {ins }}$ with the unit vector normal to the isoscalar surfaces, $n=-\nabla c /|\nabla c|$. It is evident that $\nabla \rho$ and $\nabla T_{\text {ins }}$ are apparently aligned and counter-aligned, respectively, with $\mathbf{n}$, and are therefore almost normal to the flame (see Figures 11 (a) and 11(b)). The vorticity vector, $\omega$, seems to be predominantly orthogonal to $\mathbf{n}$ within the flame for all Lewis numbers

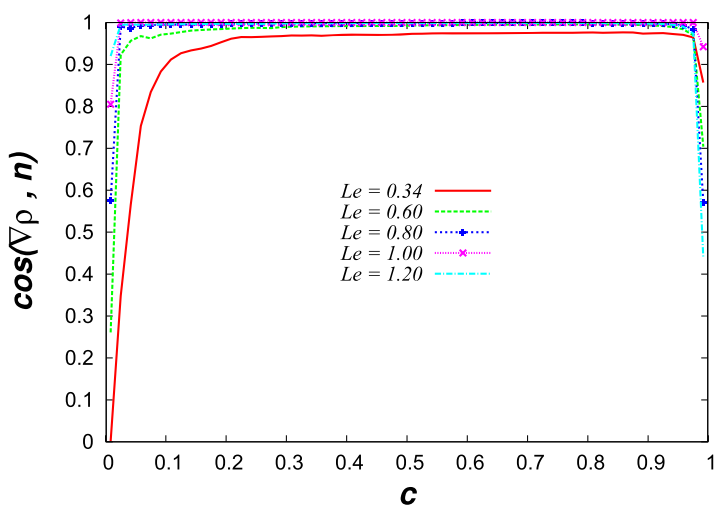

(b)

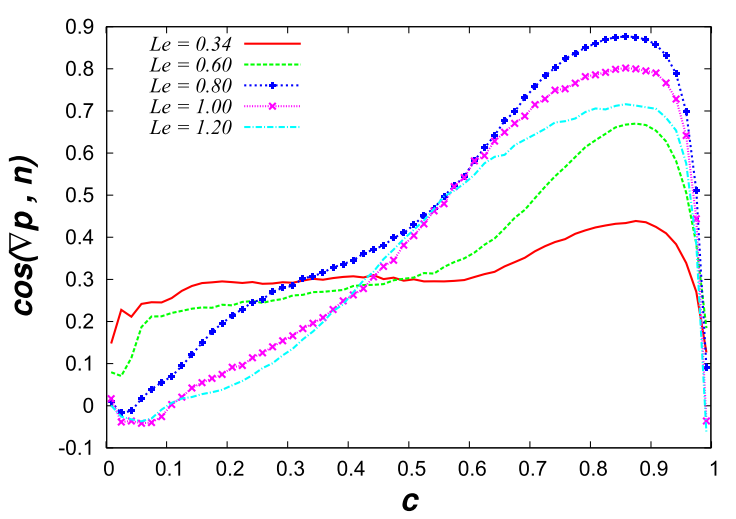

(d)

FIG. 11. Mean alignments of the unit vector, $\mathbf{n}$, normal to the iso-scalar surface $c(\mathbf{x}, t)=$ const and (a) temperature gradient, (b) density gradient, (c) vorticity vector, and (d) pressure gradient. 
(Figure 11(c)). The vectors $\nabla p$ and $\mathbf{n}$ show no preferred relative orientation (Figure 11(d)); the mean alignments of $\mathbf{n}$ and $\omega$ with respect to the eigenvectors of the strain rate tensor have been discussed elsewhere. ${ }^{38}$

For low Mach number and unity Lewis number flames, the non-dimensional temperature $T=\left(T_{\text {ins }}-T_{0}\right) /\left(T_{a d}-T_{0}\right)$ can be equated to $c$ and $\nabla \rho$ can be expressed as $\nabla \rho \approx \tau \rho^{2}|\nabla c| \mathbf{n} / \rho_{0}$, which leads to $\partial \rho / \partial x_{N}=\mathbf{n} \cdot \nabla \rho \approx-\rho \mathbf{n} \cdot \nabla T / T=\tau \rho^{2}|\nabla c| / \rho_{0}$. It was demonstrated by Chakraborty et al. ${ }^{38}$ that $\overline{|\nabla \rho|} \approx$ $\overline{\left(\partial \rho / \partial x_{N}\right)}$ because $(-\mathbf{n} \cdot \nabla T)$ remains close to $|\nabla c|$ even for $L e \neq 1.0$ flames. This suggests that $\rho^{-2} \nabla \rho \times \nabla p$ can be approximated as $\rho^{-2} \nabla \rho \times \nabla p \sim \tau|\nabla c| \mathbf{n} \times \nabla p / \rho_{0}$, which indicates that only the pressure gradient in the tangential direction to the flame $\left(\partial p / \partial x_{i}\right)_{t}=\left(\delta_{i j}-n_{i} n_{j}\right) \partial p / \partial x_{j}$ contributes to the baroclinic torque. It is worth noting that $\overline{\left.\left|(\nabla p)_{N}\right|\right)}=|\mathbf{n} \cdot \nabla p|$ is related to the flame normal acceleration, while the local pressure gradient in the tangential direction is induced by the surrounding flame wrinkles. The extent of flame wrinkling increases with decreasing $L e$. The combination of high magnitudes of $(\nabla p)_{t}$ and $\nabla \rho$ in the $L e<0.8$ flames gives rise to high values of $\rho^{-2} \nabla \rho \times \nabla p$. The directions between $\omega$ and $\rho^{-2} \nabla \rho \times \nabla p$ are independent of each other for both leading and trailing edges of the flame brush for the $L e=0.6,0.8,1.0$, and 1.2 flames. Although directions of $\omega$ and $\rho^{-2} \nabla \rho \times \nabla p$ remain completely
$L e=1.2$

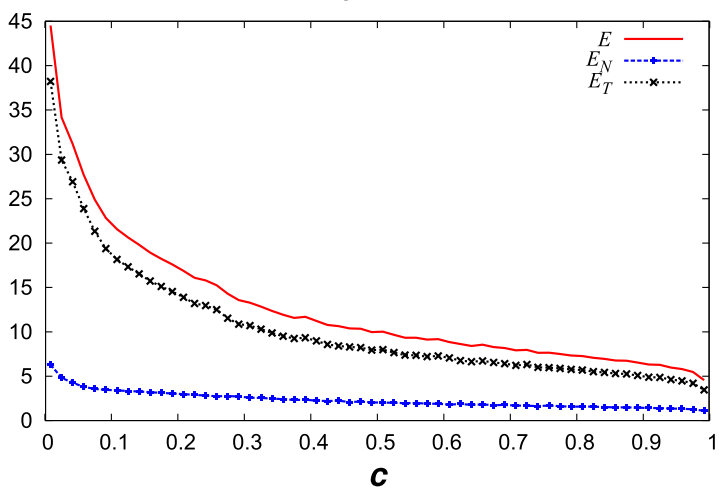

(a)

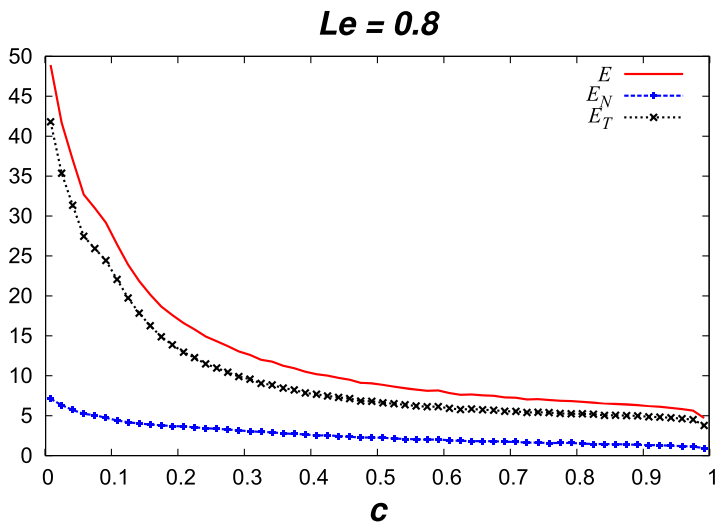

(c)
$L e=1.0$

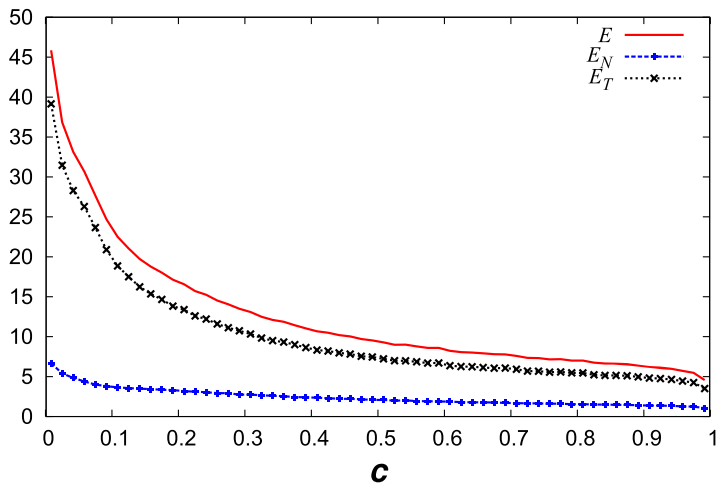

(b)

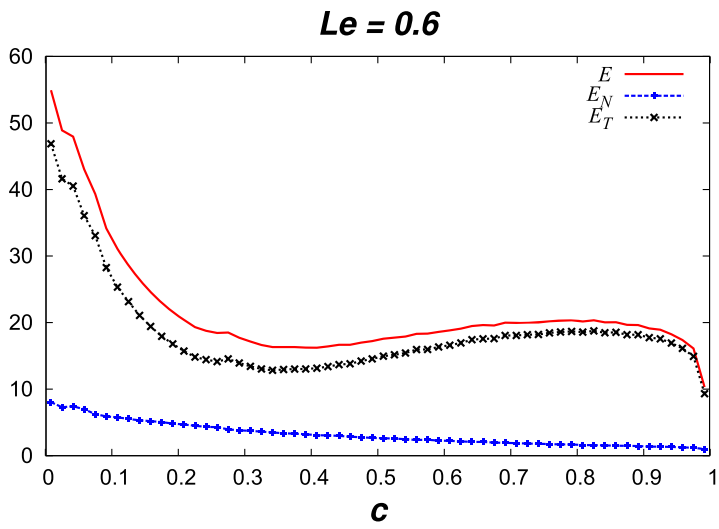

(d)

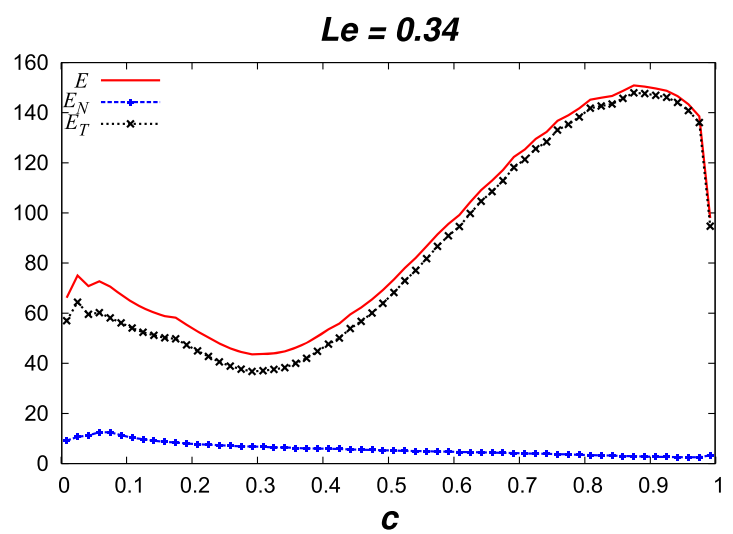

(e)

FIG. 12. Mean values of the enstrophies of the vorticity vector components tangential and normal to iso-scalar surfaces, conditional upon $c$, at different $L e$. (a) $L e=1.2$, (b) $L e=1.0$, (c) $L e=0.8$, (d) $L e=0.6$, and (e) $L e=0.34$. Variables have been normalized with $\left(\delta_{t h} / S_{L}\right)^{2}$ corresponding to the $L e=1.0$ flame. 
independent of each other for the leading edge of the flame brush, they are related on the burned gas side in the $L e=0.34$ flame due to significant density variation.

Furthermore, the relative orientation of $\rho(x, t)=$ const and $p(\mathbf{x}, t)=$ const iso-surfaces, or, equivalently, the alignment of $\nabla \rho$ and $\nabla p$, determine the magnitude of $|\sin (\nabla \rho \times \nabla p)|$. Since $\nabla p$ is given by the momentum equation and $\nabla \rho \approx|\nabla \rho| \mathbf{n}$, the baroclinic plus viscous torques can be recast as

$$
-\frac{1}{\rho^{2}} \nabla \rho \times(-\nabla p+\nabla \cdot \tau) \approx-\frac{1}{\rho}|\nabla \rho| \mathbf{n} \times \frac{D \mathbf{u}}{D t} .
$$

Therefore, the local fluid particle acceleration in the tangential direction to the flame dictates the baroclinic plus viscous torques. Fluid acceleration within the flame tends to moderately align with the inflow-outflow direction for Le equal to 0.34 and 0.6 , whereas $\mathbf{n}$ deviates from that orientation as $L e$ decreases due to flame rotation, twisting, and folding. The magnitude of the right side will thus increase as $L e$ reduces. Moreover, the baroclinic source $\left(T_{3}\right)$ and the viscous torque sink $\left(T_{4}\right)$ tend to balance each other for Le equal to the 0.8 , 1.0, and 1.2 cases (Figure 4). In any event, the viscous force torque remains a minor contributor to enstrophy production or destruction for all Lewis numbers.

The quantity $\cos (\omega, \mathbf{n})$ is expected not to vary much; Chakraborty $^{31}$ demonstrated that vorticity shows significant alignment with the eigenvector of the intermediate principal strain rate for all cases, whereas it considerably aligns with the eigenvector of the most compressive principal strain rate for the $L e=0.34$ case, this tendency increasing with decreasing Lewis number. The flame normal aligns predominantly with the eigenvector of the most extensive principal strain rate in these flames. ${ }^{38}$ Due to the orthogonality of the eigenvectors of the principal strain rates, $\cos (\omega, \mathbf{n})$ may not show much variation across the flame, which does not mean that the qualitative nature of the vorticity alignment with these local eigenvectors does not change with the Lewis number.

The analysis in this study is conducted for a single set of values of $u^{\prime} / S_{L}$ and $\tau$, for different values of Le $(0.34,0.6$,
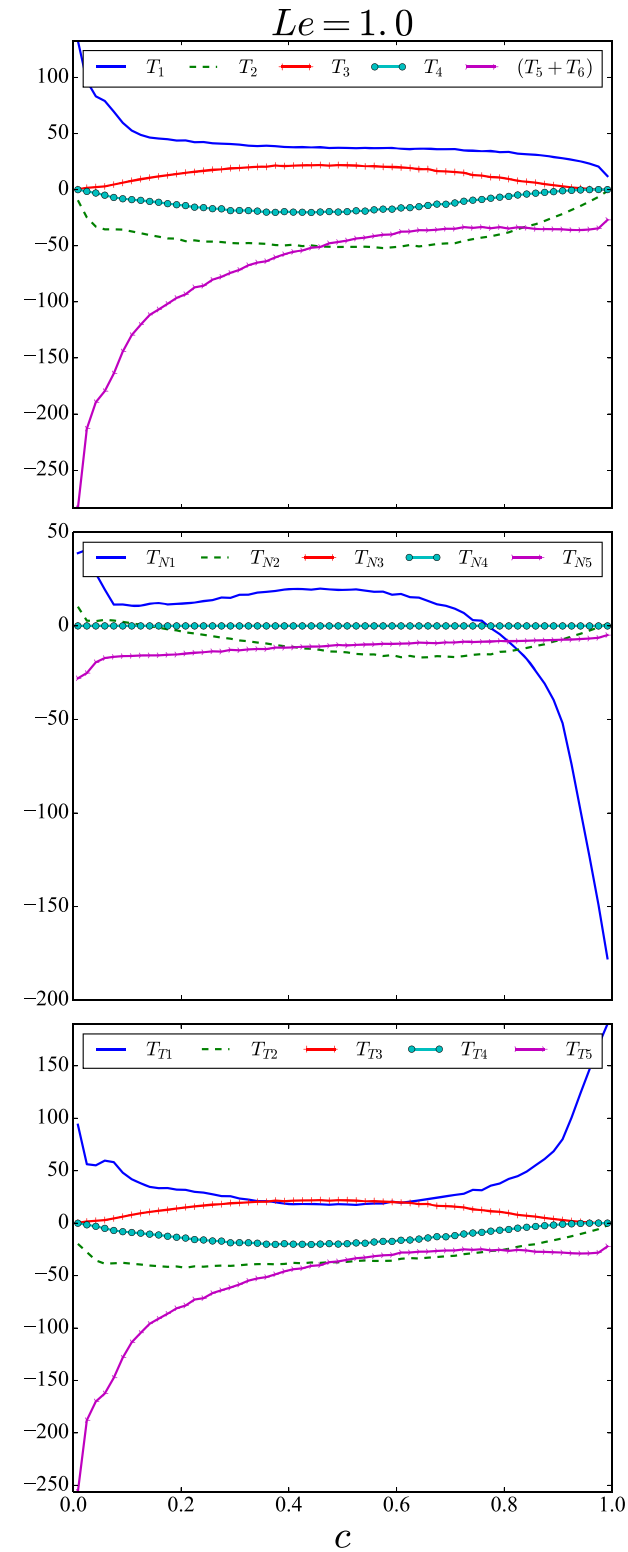

(a)
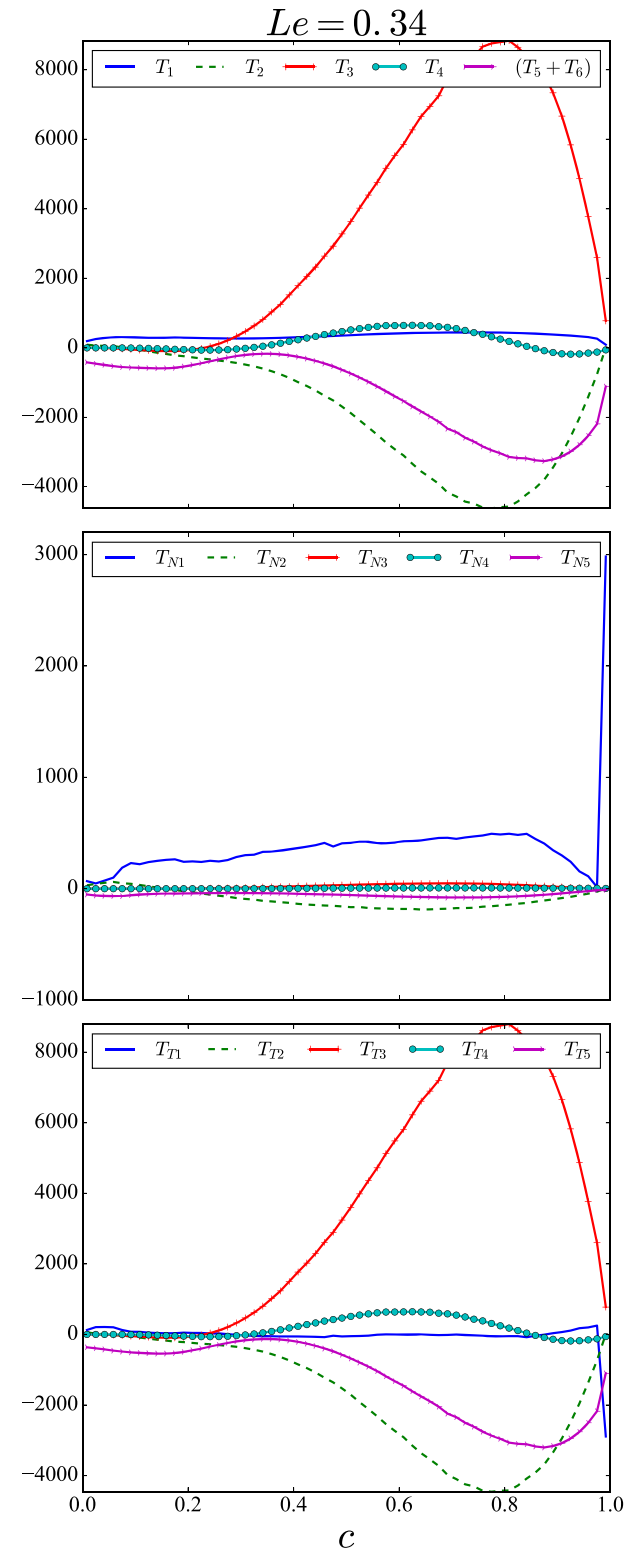

(b)
FIG. 13. Mean values of the various terms of the enstrophy and the enstrophies of the vorticity vector components tangential and normal to iso-scalar surfaces, conditional upon $c$. (a) $L e=1.0$ and (b) $L e=0.34$. Variables have been normalized with $\left(\delta_{t h} / S_{L}\right)^{3}$ corresponding to the $L e=1.0$ flame. 
$0.8,1.0$, and 1.2). For the values of $u^{\prime} / S_{L}$ and $\tau$ used here, significant enstrophy production takes place for $L e=0.34$ and 0.6 flames due to strong baroclinic vorticity generation mechanisms, whereas that contribution is relatively weak in comparison to the molecular dissipation of enstrophy for $L e=0.8,1.0$, and 1.2 cases. A modification of either $u^{\prime} / S_{L}$ or $\tau$ will modify the threshold Lewis number for which flameinduced enstrophy generation will be observed, while the strengthening of the baroclinic torque will continue to happen with decreasing $L e$.

An analysis of enstrophies of the vorticity vector components tangential and normal to iso-scalar surfaces offers an alternative perspective. Figure 12 shows that a sizable fraction of the total enstrophy is ascribable to the vorticity component tangential to iso-surfaces for all Lewis numbers and all regions of the flame. This confirms previous findings ${ }^{32}$ that the vorticity vector is predominantly a tangent to iso-scalar surfaces. Moreover, Figure 13 illustrates for $L e=1.0$ and $L e=0.34$ the contributions to $E_{T}$ and $E_{N}$ of vortex-stretching, volumetric dilatation rates, baroclinic and viscous force torques, and combined viscous transport plus dissipation. Contributions to $E_{T}$ are obtained by subtracting the corresponding terms in the transport equation for $E_{N}$ from these in the transport equation for $E$. For $L e=1.0$ (and seemingly for $L e=0.8$ and $L e=1.2$ ), the vortex-stretching term is a source of $E_{T}$ within the flame, increasing toward the trailing edge, whereas it contributes positively (negatively) to $E_{N}$ towards the unburned (burned) gas side of the flame front; vortex-stretching is the leading order contributor to $E_{N}$, but it is negligible in the $E_{T}$ transport budget. Both trends can be explained from the magnitudes of the vectors $\omega$ and $\mathbf{S} \cdot \boldsymbol{\omega}$ and alignments shown in Figures 5 and 11. Annihilation by volumetric dilatation rates for $L e=1.0$ has small negative contributions to both $E_{T}$ and $E_{N}$, while it is a major (minor) sink of $E_{T}\left(E_{N}\right)$ for $L e=0.34$; Figure 6 and expressions for the annihilation terms of $E_{N},-2\left(S_{j j}+a_{N}\right) E_{N}$ and $E_{T}$, and $-2 S_{j j} E_{T}+2 a_{N} E_{N}$, where the normal strain rate, $a_{N}>0$, help to clarify these results. Apparently, the baroclinic torque only generates $E_{T}$; this seems obvious from the previous remarks on the predominant alignment of $\nabla \rho$ and $\mathbf{n}$, which implies that the baroclinic torque contribution to $E_{N}$ in Eq. (9) is almost zero. The source of $E_{T}$ due to the baroclinic torque is moderate for $L e=1.0$ and extremely high for $L e=0.34$. For the two Lewis numbers, the viscous force torque contributes negligibly to $E_{N}$ and acts as a small sink (source) of $E_{T}$ for $L e=1.0(L e=0.34)$ within most of the flame. The combined viscous transport plus dissipation display a small negative contribution of $E_{N}$, whereas they become a leading order sink in the $E_{T}$ transport.

On the other hand, for $L e=0.34$ (and also for $L e$ $=0.6$ ), vortex-stretching leads contributions to $E_{N}$ and is negligible for $E_{T}$. Annihilation by volumetric dilatation displays a small contribution to $E_{N}$ and a large one for $E_{T}$, more pronounced in the flame zone due to heat release, and the generation of $E_{N}$ by the baroclinic torque is negligible and becomes the leading term for $E_{T}$ (for the same reasons explained for $L e \geq 0.8$ ). Viscous force torque productions of $E_{T}$ and $E_{N}$ are both negligible. The combined negative contributions to $E_{N}$ and $E_{T}$ of viscous transport and dissipation are comparable for $L e=1.0$; for $L e=0.34$, it is a leading sink of $E_{T}$, causing only small destruction of $E_{N}$.

\section{v. CONCLUSIONS}

A transport equation for the enstrophy, $E$, of a variabledensity flow of a constant dynamic viscosity fluid has been derived. An analogous equation for the enstrophy of the vorticity component normal to a given iso-scalar surface (within a flame), $E_{N}$, has also been obtained. The term by term difference between the former and the latter transport equations yields the various sources and sinks contributing to the enstrophy of the vorticity component tangential to an iso-surface, $E_{T}$. Decomposing the vorticity into its components normal and tangential to the flame clarifies the relative importance of contributions to $E$ across the flame.

The global Lewis number, $L e$, has been shown to have a profound influence on the evolution of the enstrophy field within turbulent premixed statistically planar flames. Examination of an existing DNS dataset shows that the enstrophy is significantly destroyed across the flame for $L e$ equal to 0.80 , 1.00 , and 1.20 . On the contrary, for $L e=0.60$, a slight augmentation of the enstrophy is observed within the flame, where a remarkable growth of enstrophy is apparent for $L e=0.34$.

The various terms in the enstrophy transport equation have been computed across the flame for different Lewis numbers. Vortex-stretching and viscous dissipation are the leading order contributors for the cases with Lewis numbers equal to $0.80,1.0$, and 1.20, whereas the remaining mean contributions are slightly smaller although comparable. By contrast, for the cases with Lewis numbers equal to 0.60 and 0.34 , the mean contribution of baroclinic torque is significantly greater than the other mean contributions within the flame; the mean contributions of vortex-stretching, destruction by volumetric dilatation rates and viscous transport, and dissipation remain comparable over most of the flame. The viscous torque is relatively small.

The variation with $L e$ of mean magnitudes of $\omega$ and $\mathbf{S} \cdot \omega$ and their alignment across the flame explain the behavior of the source of enstrophy by vortex-stretching. The mean value of the volumetric dilatation rate, conditional upon the reaction progress variable, accounts for changes in the enstrophy annihilation for different Lewis numbers.

An explanation for the qualitative and quantitative differences in the baroclinic torque contribution, $T_{3}$, to the enstrophy transport equation, which occurs between $L e=0.6$ and $L e$ $=0.8$, is sought in terms of the alignments of vorticity and the gradients of density and pressure, as well as their corresponding magnitudes. Positive values of $\cos (\omega, \nabla \rho \times \nabla p)$ within the flame indicate that enstrophy is always generated for all cases considered here; however, the strength of $T_{3}$ increases for $L e=0.6$ and most notably for $L e=0.34$ due to consistently higher individual values of $|\omega|,|\nabla \rho|,|\nabla p|, \sin (\nabla \rho, \nabla p)$, and $\cos (\omega, \nabla \rho \times \nabla p)$. It is found that $\nabla \rho$ is almost perfectly aligned with $\mathbf{n}$, the unit vector normal iso-scalar surfaces within the flame. The pressure gradient tangential to the flame, or, equivalently, the tangential acceleration to it, is thus responsible for the high magnitude of the baroclinic torque, which increases as $L e$ decreases due to high chemical heat release, low heat 
conduction, and significant flame wrinkling triggered by the diffusive-thermal instability.

The vorticity vector component tangential to iso-scalar surfaces is shown to be by far the major contributor to the total enstrophy. Budgets of the right sides of the enstrophy transport equations for $E_{T}$ and $E_{N}$ show that, while for $L e=1.0$, vortex-stretching, destruction by volumetric dilatation and the combination of viscous transport, and dissipation are the main contributors to $E_{N}$, all the terms are comparable within the flame for the $E_{T}$ balance. On the other hand, for $L e=0.34$, vortex stretching and annihilation by dilatation are leading contributions to $E_{N}$, whereas the baroclinic torque, annihilation by dilatation and combined viscous transport, and dissipation determine the evolution of $E_{T}$.

\section{ACKNOWLEDGMENTS}

C.D. gratefully acknowledges the support of this research by the Spanish Ministry of Economy and Competitiveness, under the CONSOLIDER-INGENIO Program, Project No. CS D2010-00011-SCORE. N.C. acknowledges N8/ARCHER for computational resources. This project has received funding from the European Union's Horizon 2020 research and innovation program under Grant Agreement No. 706672 ITPF.

${ }^{1}$ H. Tennekes and J. Lumley, A First Course in Turbulence (MIT Press, 1972).

${ }^{2}$ A. Chorin, Applied Mathematical Sciences (Springer-Verlag, 1994), Vol. 103.

${ }^{3}$ B. Karlovitz, D. Denniston, and F. Wells, J. Chem. Phys. 19, 541 (1951).

${ }^{4}$ A. Eschenroeder, Phys. Fluids 7, 1735 (1964).

${ }^{5}$ R. Günther and B. Lenze, in 14th Symposium (International) on Combustion (Elsevier, 1973), Vol. 14, p. 675.

${ }^{6}$ D. Ballal, Proc. R. Soc. A 367, 353 (1979).

${ }^{7}$ J. Driscoll and A. Gulati, Combust. Flame 72, 131 (1988).

${ }^{8}$ S. Pope, Annu. Rev. Fluid Mech. 19, 237 (1987).

${ }^{9}$ N. Chakraborty, M. Katragadda, and R. Cant, Phys. Fluids 23, 075109 (2011).

${ }^{10}$ N. Chakraborty, M. Katragadda, and R. Cant, Flow, Turbul. Combust. 87, 205-235 (2011).

${ }^{11}$ A. Steinberg, J. Driscoll, and S. Ceccio, Exp. Fluids 44, 985 (2008).

${ }^{12}$ A. Steinberg and J. F. Driscoll, Combust. Flame 156, 2285 (2009).

${ }^{13}$ A. Steinberg, J. Driscoll, and S. Ceccio, Exp. Fluids 47, 527 (2009).

${ }^{14}$ Y.-H. Im, K. Huh, S. Nishiki, and T. Hasegawa, Combust. Flame 137, 478 (2004).

${ }^{15}$ K. Nomura and S. Elghobashi, Theor. Comput. Fluid Dyn. 5, 153 (1993).

${ }^{16}$ O. Boratav, S. Elghobashi, and R. Zhong, Phys. Fluids 10(9), 2260 (1998).

${ }^{17}$ S. Elgobashi, R. Zhong, and O. Boratav, Phys. Fluids 11(10), 3123 (1999).

${ }^{18}$ F. Jaberi, D. Livescu, and C. Madnia, Phys. Fluids 12(5), 1189 (2000).

${ }^{19}$ D. Louch and K. Bray, Combust. Flame 125, 1279-1309 (2001).
${ }^{20}$ G. JaberiPatnaik and K. Kailasanath, Report No. NASA/CP-2003212376/REV1, 2003, pp. 225-228.

${ }^{21}$ S. Shanbhogue, S. Husain, and T. Lieuwen, Prog. Energy Combust. Sci. 35, 98 (2009).

${ }^{22}$ Z. Carr and D. Forliti, in 48th AIAA Aerospace Sciences Meeting (AIAA, Orlando, 2010).

${ }^{23}$ A. Lipatnikov, S. Nishiki, and T. Hasegawa, Phys. Fluids 26, 105104 (2014).

${ }^{24}$ A. Lipatnikov and J. Chomiak, Prog. Energy Combust. Sci. 36, 1 (2010).

${ }^{25}$ P. Hamlington, A. Poludnenko, and E. Oran, Phys. Fluids 23, 125111 (2011).

${ }^{26}$ T. Treurniet, F. Nieuwstadt, and B. Boersma, J. Fluid Mech. 565, 25 (2006).

${ }^{27}$ L. Cifuentes, Ph.D. dissertation (University of Zaragoza, 2015).

${ }^{28}$ L. Cifuentes, C. Dopazo, J. Martin, and C. Jimenez, Phys. Fluids 26, 065108 (2014).

${ }^{29}$ D. Haworth and T. Poinsot, J. Fluid Mech. 244, 405-436 (1992).

${ }^{30} \mathrm{C}$. Rutland and A. Trouvé, Combust. Flame 94, 41-57 (1993).

${ }^{31}$ N. Chakraborty, Eur. J. Mech.- B/Fluids 46, 201 (2014).

${ }^{32}$ N. Chakraborty, I. Konstantinou, and A. Lipatnikov, Phys. Fluids 28, 015109 (2016).

${ }^{33}$ J. J. Gorski and P. S. Bernard, Int. J. Eng. Sci. 34, 699 (1996).

${ }^{34}$ B. Bobbitt, S. Lapointe, and G. Blanquart, Phys. Fluids 28, 015101 (2016).

${ }^{35}$ B. Bobbitt and G. Blanquart, Phys. Fluids 28, 105101 (2016).

${ }^{36}$ R. Ranjan, B. Muralidharan, Y. Nagaoka, and S. Menon, Combust. Sci. Technol. 188, 1496 (2016).

${ }^{37} \mathrm{~K}$. Jenkins and R. Cant, in Recent Advances in DNS and LES, Fluid Mechanics and Its Applications Vol. 54 (Springer, 1999), pp. 191-202.

${ }^{38}$ N. Chakraborty, M. Klein, and N. Swaminathan, Proc. Combust. Inst. 32, 1409 (2009).

${ }^{39}$ N. Chakraborty and R. Cant, Combust. Flame 158, 1768-1787 (2011).

${ }^{40}$ W. T. Ashurst, N. Peters, and M. Smooke, Combust. Sci. Technol. 53, 339 (1987).

${ }^{41}$ A. Trouvé and T. Poinsot, J. Fluid Mech. 278, 1-31 (1994).

${ }^{42}$ I. Han and K. Huh, Combust. Flame 152, 194-205 (2008).

${ }^{43}$ N. Chakraborty and S. Cant, Combust. Flame 137, 129 (2004).

${ }^{44}$ N. Chakraborty, E. Hawkes, J. Chen, and R. Cant, Combust. Flame 154, 259 (2008).

${ }^{45}$ N. Chakraborty and M. Klein, Phys. Fluids 20, 065102 (2008).

${ }^{46} \mathrm{~N}$. Chakraborty and R. Cant, Phys. Fluids 17, 105105 (2005).

${ }^{47}$ Y. Gao and N. Chakraborty, Numer. Heat Transfer, Part A 69, 1201 (2016).

${ }^{48}$ Y. Gao, Y. Minamoto, M. Tanahashi, and N. Chakraborty, Combust. Sci. Technol. 188, 1398 (2016).

${ }^{49}$ N. Chakraborty, H. Kolla, R. Sankaran, E. Hawkes, J. Chen, and N. Swaminathan, Proc. Combust. Inst. 34, 1151 (2013).

${ }^{50}$ S. R. Muppala, N. K. Aluri, F. Dinkelacker, and A. Leipertz, Combust. Flame 140, 257 (2005).

${ }^{51}$ H. Kobayashi, T. Tamura, K. Maruta, T. Niioka, and F. A. Williams, in Symposium (International) on Combustion (Elsevier, 1996), Vol. 26, pp. 389-396.

${ }^{52}$ C. Law and O. Kwon, Int. J. Hydrogen Energy 29, 867 (2004).

${ }^{53}$ F. Dinkelacker, B. Manickam, and S. Muppala, Combust. Flame 158, 1742 (2011).

${ }^{54}$ T. Poinsot and S. Lele, NASA Comput. Phys. 101, 104-129 (1992).

${ }^{55}$ A. Wray, Report No. MS 202 A-1-NASA, Ames Research Center, 1990.

${ }^{56}$ T. Poinsot and D. Veynante, Theoretical and Numerical Combustion (RT Edwards, Inc., 2005).

${ }^{57}$ M. Boger, D. Veynante, H. Boughanem, and A. Trouvé, Proc. Combust. Inst. 27, 917-925 (1998).

${ }^{58}$ N. Chakraborty and R. Cant, Combust. Flame 156, 1427 (2009). 